\title{
Consistent updating of social welfare functions*
}

\author{
Takashi Hayashi ${ }^{\dagger}$
}

January 29, 2011

\begin{abstract}
It is one of the central principles in decision making that a rational agent should be forward-looking and not be bound by things already gone. We argue that this is not appealing for collective decision making, and that things already gone have necessary and substantive roles there.

We consider a dynamic process of social welfare orderings explicitly, and propose a discipline which is acceptable even after rejecting the forward-lookingness principle. It is a conjunction of two assertions: (i) the process must be dynamically consistent, which means an ex-ante welfare judgment must be respected by ex-post ones and there should be no contradiction between them; (ii) a meaningful normative postulate should be invariant under consistent updating, in the sense that a postulate met by an ex-ante welfare judgment is met by any of ex-post ones as its own property.

Based on this standpoint, we present a set of axioms for social welfare orderings which are invariant to consistent updating, and characterize a set of social welfare functions which are closed under updating. With such a class of social welfare functions, we characterize the roles for pasts and things known not to occur, which are played in the updating stage.
\end{abstract}

${ }^{*}$ I thank Chris Chambers for helpful comments and discussions.

${ }^{\dagger}$ Department of Economics, University of Texas at Austin, BRB 1.116, Austin, TX 78712. Email: th925@eco.utexas.edu. 


\section{Introduction}

\section{$1.1 \quad$ Reexamining forward-lookingness}

It is one of the central principles in decision making that a rational agent should be forwardlooking and not be bound by things already gone. It presumes that pasts and things already known not to occur are 'sunk,' and have no role to play except for the purely informational ones to facilitate inference about uncertain or strategic worlds. Throughout the paper, let us call this principle forward-lookingness. ${ }^{1}$

We argue below, however, that forward-lookingness is not an appealing principle in collective decision making, even if we accept it for individuals' evaluation of decisions, and that things already gone have necessary and substantive roles there.

Then it is natural to ask how we can discipline our argument, because one might be able to say anything if such dependence is allowed. This necessitates a question of what type of discipline should remain accepted even after rejecting forward-lookingness. This is exactly the question of the paper.

To illustrate, let us start with discussing a classical fable attributed to Aesop and one of its variations.

\section{Example 1 "The Ant and the Grasshopper (cicada in ancient versions)" An ancient version (Babrius 140 in Perry [12]):}

An ant in the winter-time was dragging out of his hole some grain which he had stored up in the summer, in order to air it. A cicada, dying of starvation, begged him to give him some of his food, to keep him alive. "What were you ding last summer?" asked the ant. "I was not loafing," said the cicada, "I was busy singing all the time." The ant laughed and barred up his grain, saying: "Dance in the winter, since you piped during the summer."

\section{A variation due to Disney (From the retelling by Brown [3]):}

.....When the ants came to the door, they found him there, half frozen. And ten of the kind and busy ants came out and carried the poor grasshopper into

\footnotetext{
${ }^{1}$ In the literature of choice under uncertainty it is called consequentialism (see for example Hammond [8] and Machina [10]), but since it is used for different meanings in different fields such as ethics, we adopt the current terminology.
} 
their house. They gave him hot corn soup. And they hurried about, making him warm.

Then the Queen of All the Ants came to him. And the grasshopper was afraid, and he begged of her: "Oh, Madam Queen, wisest of ants, please, please give me another chance."

The Queen of All the Ants looked at the poor, thin frozen grasshopper as he lay shivering there. Then she spoke these words: "With ants, just those who work may stay. So take your fiddle - and PLAY!"

The grasshopper was so happy that his foot began beating out the time in the old way, and he took up his fiddle and sang.....

To translate the above stories into a more formal setting, consider an economy with two periods, summer and winter, in which each of Ant and Grasshopper initially owns 2 units of consumption good and has an access to a storage technology, respectively. Consider that Ant saves 1 unit and Grasshopper consumes the entire 2 units during the summer. Thus in winter there is just 1 unit left which was saved by Ant. If we accept the ending of the ancient version, it reveals a judgment which ranks giving the entire 1 unit to Ant over splitting the remaining 1 unit. If we accept the ending of the modern variation, it reveals a judgment which prescribes to split the remaining 1 unit, say equally. ${ }^{234}$

Each of the judgments amounts to a problem. The second one leads to an obvious conflict between ex-post justice and ex-ante justice. The idea of ex-post justice is quite prevailing, and it is indeed regarded as a basis for redistribution in many real life situations. In this example it prescribes to split the remaining 1 unit equally. However, under the

\footnotetext{
${ }^{2}$ We consider equal division here just for simplicity.

${ }^{3}$ There may be a different interpretation of the modern variation that Ant is willing to allow redistribution, even if they are not ethically prescribed or driven to do so, because of the hope for reciprocal compensation in further future, which is a story about reputation effects. Our focus is rather on a pure ethical argument on the ex-post welfare judgment in the absence of reputation effects.

${ }^{4}$ One might give another different interpretation that the main problem here is rather whether Grasshopper is responsible for its lack or wrongness of its foresight about the food condition in winter. This will be handled as a separate issue in a later section on updating welfare judgment under uncertainty with non-common prior beliefs.
} 
natural requirement of dynamic consistency, the ex-ante welfare criterion must prescribe that Ant should receive a two-period consumption profile $(1,0.5)$ and Grasshopper should receive $(2,0.5)$, which is unfair in any sense from the ex-ante viewpoint.

The first one is, surprisingly, not supported by any of the accepted arguments in welfare economics. We should notice that the resource allocation problem they face in winter is an ex-post problem. Even if an intertemporal trade is desirable from an ex-ante viewpoint, it is a different problem, per se, whether execution of such trade is indeed desirable ex-post, since the latter ex-post problem is not about mutual exchange but typically about taking resource from somebody and giving it to somebody else. The standard welfare economics argument is based on the forward-lookingness principle and does not allow bringing up the past. How can it explain the judgment to prioritize Ant's consumption over Grasshopper's in winter, without referring to the past?

Two explanations may be attempted in the framework of welfare-based ethical argument, one is an utilitarian idea and the other is motivated by equity. The utilitarian explanation is that Ant cares for consuming in winter and Grasshopper does not. However, this comparison can make sense only in the relative way by taking another good, namely consumption in summer, into account. The right restatement of it is: Ant is willing to sacrifice consumption in summer in order to increase consumption in winter more than Grasshopper does. Then actually we are dating back to their ex-ante preferences over consumption plans and the application of ex-ante Pareto principle which would have prescribed intertemporal trading, which means we are already referring to the past.

What about explanation by means of equity? How can we say that one should get everything based on any idea of equity? The only way of rejecting equal split based on an idea of equity will be to say that it is inequitable because then Ant is receiving a twoperiod consumption profile $(1,0.5)$ and Grasshopper is receiving $(2,0.5)$. This is obviously bringing up the past and the notion of ex-ante equity.

It is hard to explain the first judgment without bringing up the past. Not only that, it is known to be indeed impossible. Zuber [14] shows that social preference over streams of social outcomes which is dynamically consistent and respects the Pareto principle can be independent of history and chronology only if all individuals have the same time preference and the aggregation rule is additive, which excludes equity concern. This is confirmed in the current paper as well. 
What is the reasonable way of making ex-post welfare judgment then? What type of discipline should remain accepted even after rejecting forward-lookingness?

One may think of simply applying the welfare criterion which was accepted ex-ante. For example, under the rationality assumption that both Ant and Grasshopper have correct foresight on the consequence of their (non-)saving, one may conclude that Ant preferred a two-period consumption profile $(1,1)$ to $(2,0)$ ex-ante and opposite for Grasshopper, and deduce that since they have unanimously agreed to a plan, Ant receiving $(1,1)$ and Grasshopper receiving $(2,0)$, it is unreasonable to redistribute consumption in the winter.

However, if we support execution of a plan only for the reason that it was accepted to be desirable in the past, where is any thinking in the present? If all what we should do in the present is just to follow the prescription by a judgment made in the past, we are no more than a 'slave' of the past. A practical argument may say that execution of an ex-ante agreed plan must be enforcing (or self-enforcing) even if there is no substantive reason to do so ex-post, because otherwise such a plan is null and never be implemented. For example, it says that you should pay your debt because otherwise you are punished or nobody finances you in the beginning. Our argument is rather that fulfillment of a plan is not only practically necessary but also there should be a welfare-based ethical reason for it. That is, there should be an ethical reason for why you should pay your debt. ${ }^{5}$

Being consistent over time requires that our ex-post welfare judgment must coincide with the 'projection' of the ex-ante one, and such projection can be taken in an obvious manner. To illustrate schematically, let $a$ and $b$ denote possible social outcomes in period 1 and $x$ and $y$ denote possible outcomes in period 2. Consider that an ex-ante social ranking $\succsim$ yields let's say $(a, x) \succ(b, y) \succ(b, x) \succ(a, y)$. Then the ex-post ranking conditional on $a$, denoted $\succsim_{a}$ may be simply taken as $x \succ_{a} y$, which is the projection of $(a, x) \succ(a, y)$. Similarly the ex-post ranking $\succsim_{b}$ conditional on $b$ is taken to be $y \succ_{b} x$. However, even if the ex-ante ranking $\succsim$ possesses normatively appealing properties, it is a different problem if the ex-post rankings $\succsim_{a}$ and $\succsim_{b}$ have such properties.

There should be a distinction between structure of welfare judgment, the form of thinking, and particular choice of welfare weights and priorities. We view that a normatively meaningful structure which appears in the ex-post welfare judgment should appear again

\footnotetext{
${ }^{5}$ This is different from an ethical viewpoint that fulfilling a promise or prior agreement is a virtue by itself regardless of its welfare consequence.
} 
in the ex-post welfare judgment as its own independent property and at the same time in an invariant manner, while particular weights should depend on histories and vary over time. This is the discipline what we mean.

Let us further illustrate our points with a different situation, risk and uncertainty.

\section{Example 2 "Machina's Mom" \\ From Machina (1989):}

Mom has a single indivisible item — a "treat" — which she can give to either daughter Abigail or son Benjamin. Assume that she is indifferent between Abigail getting the treat and Benjamin getting the treat, and strongly prefers either of these outcomes to the case where neither child gets it. However, in a violation of the precepts of expected utility theory, Mom strictly prefers a coin flip over either of these sure outcomes, and in particular, strictly prefers $1 / 2$ : $1 / 2$ to any other pair of probabilities. This random allocation procedure would be straightforward, except that Benjie, who cut his teeth on Raiffa's classic Decision Analysis, behaves as follows:

Before the coin is flipped, he requests a confirmation from Mom that, yes, she does strictly prefer a 50:50 lottery over giving the treat to Abigail. He gets her to put this in writing. Had he won the flip, he would have claimed the treat. As it turns out, he loses the flip. But as Mom is about to give the treat to Abigail, he reminds Mom of her "preference for flipping a coin over giving it to Abigail (producing her signed statement), and demands that she flip again.

What would your Mom do if you tried to pull a stunt like this? She would undoubtedly say "You had your chance!" and refuse to flip the coin again. This is precisely what Mom does.

Machina continues, "By replying "You had your chance," Mom is reminding Benjamin of the existence of the snipped-off branch (the original $1 / 2$ probability of $B$ ) and that her preferences are not separable, so the fact that nature could have gone down that branch still 
matters. Mom is rejecting the property of consequentialism - and, in my opinion, rightly so." Here the term 'consequentialism' is what the forward-lookingness principle is called in the literature of choice under uncertainty, which in particular says that the decision should be independent of events or outcomes which turned out not to occur (see Hammond [8] for example).

Again, each of Mom's claim and Benjamin's claim amounts to a problem. If Mom's claim to reject consequentialism is no more than mechanically following the ex-ante welfare judgment, our thinking in the present does not possess any normative feature which is appealing by itself. In general, a meaningful property of non-expected utility preference might not be preserved through taking projection, while all the axioms of expected utility theory and additive aggregation are known to be maintained after consequentialist updating. On the other hand, if we accept Benjamin's claim, as far as we want our welfare judgment to be dynamically consistent, it leads to that the en-ante judgment must support Abigail winning the item with probability $1 / 2 \times 1 / 2=1 / 4$ and Benjamin winning with probability $1 / 2+1 / 2 \times 1 / 2=3 / 4$, which is unfair in any sense from the ex-ante viewpoint.

The example suggests that a consistent balance between ex-ante and ex-post equity is impossible if we don't take non-occurred events and outcomes into account. Indeed it is shown to be impossible by Hammond [8]. How should non-occurred events and outcomes be taken into account, then? Here as well, we propose a discipline that a normatively meaningful structure which appears in the ex-post welfare judgment should appear again in the ex-post welfare judgment as its own independent property and at the same time in an invariant manner, while particular weights should depend on non-occurred events and outcomes and vary across events.

\subsection{Outline}

In this paper we consider a dynamic process of social welfare orderings explicitly. We then propose a discipline, which is a conjunction of two assertions: (i) the process must be dynamically consistent, which means an ex-ante welfare judgment must be respected by expost ones and there should be no contradiction between them; (ii) a meaningful normative postulate should be invariant under consistent updating, in the sense that a postulate met by an ex-ante welfare judgment is met by any of ex-post ones as its own property.

Based on this standpoint, we present a set of axioms for social welfare orderings which 
are invariant to consistent updating, and characterize a set of social welfare functions which are closed under updating. With such a class of social welfare functions, we characterize the roles for pasts and things known not to occur, which are played in the updating stage.

The problem studied here lies almost everywhere in our life: imagine any situation that there is no prior contract or agreement explicitly made in the past, and one goes to the court requesting compensation for a loss or lack of fairness due to somebody's wrongdoing or events for which she is not responsible; or imagine any situation that a prior contract or agreement is violated by somebody and one goes to the court requesting the fulfillment of it or compensation for the foregone benefit due to the violation. Such situation is almost everywhere. Requesting compensation or fulfillment of a contract is nothing but bringing up the past. The court then needs to make a reasoning not as a slave of the past, but as an independent thinker, while it needs to track back the history in order to calculate the right compensation, by investigating what could have been done, what could have occurred and what had been conceived as agreeable. Our aim is to model such reasoning.

After concluding the introduction with discussion on related literature, the paper proceeds as follows. In Section 2, we present an axiomatic characterization of a class of static social welfare functions, which allows for the role of equity concern and is shown in later sections to be closed under consistent updating. In Section 3, we consider the problem of updating the deterministic setting with intertemporal compensations, and propose a condition that an axiom imposed on social welfare ordering be preserved under consistent updating. Then we show that the set of axioms as proposed in Section 2, hence the class of characterized social welfare functions, is closed under consistent updating, and present the updating rule of welfare weights and equity attitudes, which explains the role of pasts In Section 4, we consider the problem of updating along with resolution of uncertainty, and propose a condition that an axiom imposed on social welfare ordering be preserved under consistent updating. Then we show that the the set of axioms as proposed in Section 2 , hence the class of characterized social welfare functions, is closed under updating, and present the updating rule of welfare weights and equity attitudes, which explains the role of things known not to occur. 


\subsection{Related Literature}

Zuber [14] shows that social preference over deterministic streams of outcomes respecting the Pareto principle is dynamically consistent and independent of histories and time, which together imply stationarity, only if individual preferences are stationary additive with discount factors being identical across individuals and the aggregation rule is additive. This means that dynamically consistent and history-time independent social preference does not allow for any role of equity concern, or even the role of intertemporal compensation and trades based on the difference of time preferences.

Hammond [8] shows in the setting of uncertainty that social choice rule respecting the Pareto principle is dynamically consistent and follows consequentialism only if it maximizes a weighted sum of individual expected utilities. This strengthens the result by Harsanyi [9] that a list of social preference and individual preferences having the expected utility forms respects the Pareto principle only if the social expected utility is a weighted sum of individual expected utilities. See also Bommier and Zuber [2] for a related result. This means that dynamically consistent and consequentialist social choice rule does not allow for the role of equity concern as considered in Diamond [6], that the society should have a strict preference for randomization for the sake of ex-ante fairness. As cited above, Machina [10] discusses the incompatibility between such equity concern and consequentialism.

In the setting of risk (objective uncertainty), Epstein and Segal [7] axiomatically characterize the class of quadratic social welfare functions, which allows the room for the Diamond-type equity concern. There Epstein and Segal discuss 'dynamic consistency' of their model itself, arguing that a non-consequentialist update of a quadratic social welfare function is again quadratic. The current research, although leading to a different class of social welfare functions, is viewed as going further on this direction in the sense that we set consistent updating explicitly as the main issue and investigate its full implication in a more general setting with possibly heterogeneous beliefs.

\section{The class of static social welfare functions}

\subsection{Basic axioms and characterizations}

This section presents a set of axioms, which in later sections are translated into dynamic setting and shown to be preserved under consistent updating along with time passing and 
resolution of uncertainty, and presents a class of social welfare functions characterized by that, which is shown to be closed under consistent updating.

Let $I$ be the set of individuals. For technical reason, we assume $|I| \geq 3$. We assume that individual utilities to are given as cardinal and interpersonally comparable objects. Let $\mathbb{R}^{I}$ be the domain of such individual utilities. We consider a social welfare ordering $\succsim$ defined over $\mathbb{R}^{I}$.

We consider the following axioms.

Order: $\succsim$ is complete and transitive.

Continuity: $\succsim$ is a closed subset of $\mathbb{R}^{I} \times \mathbb{R}^{I}$.

Pareto: for all $U, V \in \mathbb{R}^{I}, U_{i} \geq V_{i}$ for every $i \in I$ implies $U \succsim V$, and the conclusion is strict additionally if $U_{i}>V_{i}$ for some $i \in I$.

Inequality Aversion: for all $U, V \in \mathbb{R}^{I}$ and $c \in[0,1], U \sim V$ implies $c U+(1-c) V \succsim U$.

Separability: for all $J \subset I$, for all $U_{J}, V_{J} \in \mathbb{R}^{J}$ and $U_{-J}, V_{-J} \in \mathbb{R}^{I \backslash J},\left(U_{J}, U_{-J}\right) \succsim$ $\left(V_{J}, U_{-J}\right)$ holds if and only if $\left(U_{J}, V_{-J}\right) \succsim\left(V_{J}, V_{-J}\right)$.

Shift Covariance: for all $U, V \in \mathbb{R}^{I}$ and $c \in \mathbb{R}, U \succsim V$ implies $U+c \mathbf{1} \succsim V+c \mathbf{1}$.

The last Shift Covariance axiom is concerned with interpersonal comparison of utilities, and saying that adding 'equal utility' to everybody does not change the social welfare ranking. This means that attitude toward inequality is independent of the absolute level of utilities.

Let $\Delta(I)=\left\{a \in \mathbb{R}_{+}^{I}: \sum_{i \in I} a_{i}=1\right\}$ be the set of normalized weights over individuals which add up to 1 , and let $\operatorname{int} \Delta(I)=\Delta(I) \cap \mathbb{R}_{++}^{I}$ be the set of positive weight vectors.

Theorem 1 A social welfare ordering $\succsim$ satisfies Order, Continuity, Pareto, Inequality Aversion, Separability and Shift Covariance if and only if either of the following two cases holds:

(i) there exist a vector $a \in$ int $\Delta(I)$ and a number $\lambda>0$ such that $\succsim$ is represented in the form

$$
\Phi(U)=-\sum_{i \in I} a_{i} e^{-\lambda U_{i}}
$$


We call this class of orderings and representations exponential class.

(ii) there exists a vector $a \in$ int $\Delta(I)$ such that $\succsim$ is represented in the form

$$
\Phi(U)=\sum_{i \in I} a_{i} U_{i}
$$

We call this class of orderings and representations additive class.

Moreover, in case (i) $a$ and $\lambda$ are unique and in case (ii) $a$ is unique.

Proof. See appendix.

We will also consider a weaker version of Shift Covariance. This is because 'equality' does not necessarily mean 'equality of utilities,' depending on situations. This is particularly the case when 'scaling' of utility is different across individuals. As we will see later, when individuals' subjective weights on future differ it may be natural to say that their scalings of future utilities are treated as different too, and when individuals' beliefs on events differ it may be natural to say that their scalings of utilities contingent on events are treated as different too. The axiom below says that up to some scalings adding 'equal condition' to everybody does not change the social welfare ranking.

General Shift Covariance: there exists $W \in \mathbb{R}_{++}^{I}$ such that for all $U, V \in \mathbb{R}^{I}$ and $c \in \mathbb{R}, U \succsim V$ implies $U+c W \succsim V+c W$.

Theorem 2 A social welfare ordering $\succsim$ satisfies Order, Continuity, Pareto, General Shift Covariance and Separability if and only if either of the following two cases holds:

(i) there exists a vector $a \in \operatorname{int} \Delta(I)$ and a vector $\lambda \in \mathbb{R}_{++}^{I}$ such that $\succsim$ is represented in the form

$$
\Phi(U)=-\sum_{i \in I} a_{i} e^{-\lambda_{i} U_{i}}
$$

We call this class of orderings and representations generalized exponential class.

(ii) there exists a vector $a \in \operatorname{int} \Delta(I)$ such that $\succsim$ is represented in the form

$$
\Phi(U)=\sum_{i \in I} a_{i} U_{i}
$$

We again call this class of orderings and representations additive class.

Moreover, in case (i) $a$ and $\lambda$ are unique and in case (ii) $a$ is unique.

Proof. See appendix. 


\subsection{Comparative inequality aversion}

Here we discuss the normative content of the parameters in the social welfare function characterized above. For the exponential class and additive class, one can make a straightforward interpretation of the parameters, which is an analogue of the standard argument on risk aversion: $a$ explains welfare weights on individuals and $\lambda$ explains the degree of inequality aversion.

We extend this interpretation to the generalized exponential class, in which the notion of 'equality' may depend on the difference of scalings of utilities across individuals and hence the degree of inequality aversion may be different across individuals.

We we define comparative inequality aversion in the following way.

Definition $1 \succsim$ is more inequality averse than $\succsim^{\prime}$ if there exists a vector $W \in \mathbb{R}_{++}^{I}$ such that for all $c \in \mathbb{R}$ and $U \in \mathbb{R}^{I}, U \succsim c W$ implies $U \succsim^{\prime} c W$.

Here the ray spanned by vector $W$ reflects what is regarded as 'equal' by the given social welfare judgment. This includes the standard definition of inequality aversion as a special case that $W$ is proportional to 1 .

Theorem 3 Suppose $\succsim$ and $\succsim^{\prime}$ fall in the generalized exponential class, where $(a, \lambda)$ describes $\succsim$ and $\left(a^{\prime}, \lambda^{\prime}\right)$ describes $\succsim^{\prime}$, respectively. Then $\succsim$ is more inequality averse than $\succsim^{\prime}$ if and only if $a=a^{\prime}$ and $\lambda=\mu \lambda^{\prime}$ for some $\mu \geq 1$.

Proof. 'If' part is routine. We prove the 'only if' part.

Consider the 'marginal rate of substitution' between individual utilities associated with $\succsim$, which is given by

$$
M R S(U)=\left(\frac{\lambda_{i} a_{i} e^{-\lambda_{i} U_{i}}}{\lambda_{1} a_{1} e^{-\lambda_{1} U_{1}}}\right)_{i \in I \backslash\{1\}}
$$

Note that

$$
M R S(c W)=\left(\frac{\lambda_{i} a_{i}}{\lambda_{1} a_{1}}\right)_{i \in I \backslash\{1\}}
$$

for all $c$, where $W=\left(1 / \lambda_{i}\right)_{i \in I}$. Do the same argument for $M R S^{\prime}$ and $W^{\prime}=\left(1 / \lambda_{i}^{\prime}\right)_{i \in I}$ associated with $\succsim^{\prime}$.

For $\succsim$ and $\succsim^{\prime}$ to be comparable, it must be that $W$ and $W^{\prime}$ span the same ray passing through the origin. Therefore, $\lambda=\mu \lambda^{\prime}$ for some $\mu>0$. Since $M R S$ and $M R S^{\prime}$ must be the same along the ray, we have $a=a^{\prime}$. By comparing the second-order derivatives, we obtain $\mu \geq 1$. 
Now it is immediate to see the following claim.

Corollary 1 Suppose $\succsim$ and $\succsim^{\prime}$ fall in the exponential class, where $(a, \lambda)$ describes $\succsim$ and $\left(a^{\prime}, \lambda^{\prime}\right)$ describes $\succsim^{\prime}$, respectively. Then $\succsim$ is more inequality averse than $\succsim^{\prime}$ if and only if $a=a^{\prime}$ and $\lambda \geq \lambda^{\prime}$.

\section{Consistent updating with intertemporal compensa- tions}

\subsection{Consistent updating under homogeneous discounting}

In this section, we demonstrate how the axioms imposed on static social welfare functions are preserved under consistent updating with intertemporal compensation in a deterministic setting. Here we limit attention to the case that discounting is homogeneous across individuals and we will extend the argument to the case of heterogeneous discounting in a later section.

Consider a discrete and infinite time horizon. Given $t$, let $u_{t}=\left(u_{i t}\right)_{i \in I}$ be the list of utilities received by the individuals at period $t$. Given $t$, let $\mathbf{u}^{t-1}=\left(u_{0}, \cdots, u_{t-1}\right)$ be a history of utilities received by the individuals before period $t$. Denote the initial point with null history by $\emptyset$. Also, given $t$, let $L^{I \times[t, \infty]}=\left\{\mathbf{u}_{t}=\left(u_{t}, u_{t+1}, \cdots\right): \max _{i} \sup _{\tau \geq t}\left|u_{i \tau}\right|<\infty\right\}$ be the set of bonded sequences starting at period $t$. For each possible history $\mathbf{u}^{t-1}$, let $\succsim_{\mathbf{u}^{t-1}}$ be the social welfare ordering over $L^{I \times[t, \infty]}$ conditional on $\mathbf{u}^{t-1}$. Given $\mathbf{u}_{t}, \mathbf{v}_{t} \in L^{I \times[t, \infty]}$, the ranking may be written for example as $\mathbf{u}_{t}{\succsim \mathbf{u}^{t-1}}_{\mathbf{v}_{t}}$. Let $\left\{\succsim_{\mathbf{u}^{t-1}}\right\}$ be a process of such social welfare orderings. Also, let $\beta \in(0,1)$ be the discount factor which is assumed to be common across the individuals.

We will consider two kinds of axioms. One is properties of social welfare criteria which are desirable to be satisfied at each time in each possible history, and are investigated if they are preserved under consistent updating across times. We call such axioms intraprofile axioms. The other is about relationships between social welfare orderings indexed with different times and histories. We call such axioms inter-profile axioms.

Dynamic consistency condition is the inter-profile axiom we impose throughout the analysis. 
Dynamic Consistency: for all $t$ and $\mathbf{u}^{t-1}$, for all $u_{t} \in \mathbb{R}^{I}$ and $\mathbf{u}_{t+1}, \mathbf{v}_{t+1} \in L^{I \times[t, \infty]}$,

$$
\left(u_{t}, \mathbf{u}_{t+1}\right) \succsim_{\mathbf{u}^{t-1}}\left(u_{t}, \mathbf{v}_{t+1}\right) \quad \Longleftrightarrow \mathbf{u}_{t+1} \succsim_{\left(\mathbf{u}^{t-1}, u_{t}\right)} \mathbf{v}_{t+1}
$$

We define the condition that an intra-profile axiom is preserved under consistent updating across times.

Definition 2 An intra-profile axiom is said to be preserved under consistent updating if for any process of social welfare orderings $\left\{\succsim_{\mathbf{u}^{t-1}}\right\}$ satisfying Dynamic Consistency, for all $\mathbf{u}^{t-1}$ and $u_{t} \in \mathbb{R}^{I}$, if it being stated on $\succsim_{\mathbf{u}^{t-1}}$ is true then it being stated on $\succsim_{\mathbf{u}^{t-1}, u_{t}}$ is true.

Example 3 The following intra-profile axioms are not preserved under consistent updating.

Anonymity at a given history $\mathbf{u}^{\mathbf{t}-\mathbf{1}}$ : for all $\mathbf{u}_{t}, \mathbf{v}_{t} \in L^{I \times[t, \infty]}$ and any permutation $\pi$ over $I$

$$
\mathbf{u}_{t} \succsim_{\mathbf{u}^{t-1}} \mathbf{v}_{t} \Longleftrightarrow \mathbf{u}_{t}^{\pi} \succsim_{\mathbf{u}^{t-1}} \mathbf{v}_{t}^{\pi}
$$

Homogeneity at a given history $\mathbf{u}^{\mathbf{t}-\mathbf{1}}$ : for all $\mathbf{u}_{t}, \mathbf{v}_{t} \in L^{I \times[t, \infty]}$ and for all non-negative number $c$,

$$
\mathbf{u}_{t}{\mathbf{u}^{t-1}} \mathbf{v}_{t} \Longleftrightarrow c \mathbf{u}_{t}{\mathbf{u}^{t-1}} c \mathbf{v}_{t}
$$

To see that these axioms are not preserved under updating, consider the social welfare function at history $\mathbf{u}^{t-1}$ in the form

$$
\Phi\left(\mathbf{u}_{t} \mid \mathbf{u}^{t-1}\right)=\min _{i \in I} \sum_{\tau=t}^{\infty} \beta^{\tau-t} u_{i \tau} .
$$

This obviously satisfies Anonymity and Homogeneity at History $\mathbf{u}^{t-1}$. However, its consistent updating $\Phi\left(\mathbf{u}_{t+1} \mid \mathbf{u}^{t-1}, u_{t}\right)$ at history $\left(\mathbf{u}^{t-1}, u_{t}\right)$ must be ordinally equivalent to the function

$$
\min _{i \in I}\left(u_{i t}+\beta \sum_{\tau=t+1}^{\infty} \beta^{\tau-(t+1)} u_{i \tau}\right),
$$

which does not satisfy Anonymity or Homogeneity as the ranking over $\mathbf{u}_{t+1}$.

The reason for this is rather easier to see at a conceptual level. Note that these axioms are about renaming and rescaling future utilities. Anonymity says that identity of individuals does not matter in evaluating future utilities. However, in the presence of past 
outcomes which have given different utilities to different individuals, identity of the individuals is indispensable in consistent updating. Homogeneity says that changing the common scale of individual utilities in the future does not matter. However, changing the scale of future utilities changes the impact of past utilities, which in general results in violating dynamic consistency.

To introduce intra-profile axioms, fix an arbitrarily time $t$ and history $\mathbf{u}^{t-1}$. The first one is self-explanatory.

Order: $\succsim_{\mathbf{u}^{t-1}}$ is complete and transitive ordering over $L^{I \times[t, \infty]}$.

Second we consider a group-separability axiom.

Separability: for all $J \subset I$, for all $\mathbf{u}_{J t}, \mathbf{v}_{J t} \in L^{J \times[t, \infty]}$ and for all $\mathbf{u}_{-J t}, \mathbf{v}_{-J t} \in L^{(I \backslash J) \times[0, \infty]}$, $\left(\mathbf{u}_{J t}, \mathbf{u}_{-J t}\right) \succsim_{\mathbf{u}^{t-1}}\left(\mathbf{v}_{J t}, \mathbf{u}_{-J t}\right)$ holds if and only if $\left(\mathbf{u}_{J t}, \mathbf{v}_{-J t}\right) \succsim_{\mathbf{u}^{t-1}}\left(\mathbf{v}_{J t}, \mathbf{v}_{-J t}\right)$.

Next we consider an axiom that the social ranking over utility streams depends only on individuals' discounted utility evaluations. This says that each individual is taken to be maintaining 'unity' over time, and her utilities at different periods are summarized by herself, and the social ranking concerns only such summaries by the individuals. One may see the non-triviality of this condition by thinking of a situation in which each individual has different 'selves' at different periods and there is a conflict among them, and the social choice may have to concern resolution of such conflicts as well.

DU-Pareto: for all $\mathbf{u}_{t}, \mathbf{v}_{t} \in L^{I \times[t, \infty]}, \sum_{\tau=t}^{\infty} \beta^{\tau-t} u_{i \tau} \geq \sum_{\tau=t}^{\infty} \beta^{\tau-t} v_{i \tau}$ for all $i \in I$ implies $\mathbf{u}_{t} \succsim_{\mathbf{u}^{t-1}} \mathbf{v}_{t}$, and the conclusion is strict additionally if $\sum_{\tau=t}^{\infty} \beta^{\tau-t} u_{i \tau}>\sum_{\tau=t}^{\infty} \beta^{\tau-t} v_{i \tau}$ for some $i \in I$.

Under DU-Pareto, one can define a social ranking induced over discounted utilities.

Definition 3 Given $t$ and $\mathbf{u}^{t-1}$, the DU-welfare ordering $\succsim_{\mathbf{u}^{t-1}}^{*}$ over $\mathbb{R}^{I}$ induced by $\succsim_{\mathbf{u}^{t-1}}$ is defined by

$$
U_{t} \succsim_{\mathbf{u}^{t-1}}^{*} V_{t} \Longleftrightarrow \mathbf{u}_{t} \succsim_{\mathbf{u}^{t-1}} \mathbf{v}_{t}
$$

for $\mathbf{u}_{t}, \mathbf{v}_{t} \in L^{I \times \infty}$ with $U_{t}=\sum_{\tau=t}^{\infty} \beta^{\tau-t} u_{i \tau}$ and $V_{t}=\sum_{\tau=t}^{\infty} \beta^{\tau-t} v_{i \tau}$ respectively. The DUPareto axiom ensures that this is well-defined. 
We consider the following three intra-profile axioms on the induced ranking. Although one can write them down directly in terms of the original ranking, this helps simplifying the exposition.

DU-Continuity: $\succsim_{\mathbf{u}^{t-1}}^{*}$ is a closed subset of $\mathbb{R}^{I} \times \mathbb{R}^{I}$.

DU-Inequality Aversion: for all $U_{t}, V_{t} \in \mathbb{R}^{I}$ and $c \in[0,1], U_{t} \sim_{\mathbf{u}^{t-1}}^{*} V_{t}$ implies $c U_{t}+$ $(1-c) V_{t} \succsim_{\mathbf{u}^{t-1}}^{*} U_{t}$.

DU-Shift Covariance: for all $U_{t}, V_{t} \in \mathbb{R}^{I}$ and $c \in \mathbb{R}, U_{t} \succsim_{\mathbf{u}^{t-1}}^{*} V_{t}$ implies $U_{t}+c \mathbf{1} \succsim_{\mathbf{u}^{t-1}}^{*}$ $V_{t}+c \mathbf{1}$.

Proposition 1 Order, Separability, DU-Pareto, DU-Continuity, DU-Inequality Aversion and DU-Shift Covariance are preserved under consistent updating.

\section{Proof.}

Order: Obvious.

Separability: Let $\left(\mathbf{u}_{J, t+1}, \mathbf{u}_{-J, t+1}\right) \succsim_{\mathbf{u}^{t-1}, u_{t}}\left(\mathbf{v}_{J, t+1}, \mathbf{u}_{-J, t+1}\right)$. By Dynamic Consistency, this holds if and only if $\left(\left(u_{J t}, \mathbf{u}_{J t}\right),\left(u_{-J t}, \mathbf{u}_{-J t}\right)\right) \succsim_{\mathbf{u}^{t-1}}\left(\left(u_{J t}, \mathbf{v}_{J t}\right),\left(u_{-J t}, \mathbf{u}_{-J t}\right)\right)$. By Separability assumed for $\succsim_{\mathbf{u}^{t-1}}$, this holds if and only if $\left(\left(u_{J t}, \mathbf{u}_{J t}\right),\left(u_{-J t}, \mathbf{v}_{-J t}\right)\right) \succsim_{\mathbf{u}^{t-1}}\left(\left(u_{J t}, \mathbf{v}_{J t}\right),\left(u_{-J t}, \mathbf{v}_{-J t}\right)\right)$. By Dynamic Consistency, this holds if and only if $\left(\mathbf{u}_{J, t+1}, \mathbf{v}_{-J, t+1}\right) \succsim_{\mathbf{u}^{t-1}, u_{t}}\left(\mathbf{v}_{J, t+1}, \mathbf{v}_{-J, t+1}\right)$. DU-Pareto: Let $\sum_{\tau=t+1}^{\infty} \beta^{\tau-t} u_{i \tau} \geq \sum_{\tau=t+1}^{\infty} \beta^{\tau-t} v_{i \tau}$ for all $i \in I$. Then $u_{i t}+\sum_{\tau=t}^{\infty} \beta^{\tau-t} u_{i \tau} \geq$ $u_{i t}+\sum_{\tau=t}^{\infty} \beta^{\tau-t} v_{i \tau}$ for all $i \in I$. By DU-Pareto assumed for $\succsim_{\mathbf{u}^{t-1}}$, we have $\left(u_{t}, \mathbf{u}_{t+1}\right) \succsim_{\mathbf{u}^{t-1}}$ $\left(u_{t}, \mathbf{v}_{t+1}\right)$. By Dynamic Consistency, $\mathbf{u}_{t+1} \succsim_{\left(\mathbf{u}^{t-1}, u_{t}\right)} \mathbf{v}_{t+1}$. The strict case is proved similarly. DU-Continuity: Suppose $U_{t+1}^{\nu} \succsim_{\mathbf{u}^{t-1}, u_{t}}^{*} V_{t+1}^{\nu}$ for all $\nu$. By Dynamic Consistency, we have $\left(u_{i t}+\beta U_{i, t+1}^{\nu}\right)_{i \in I} \succsim_{\mathbf{u}^{t-1}}^{*}\left(u_{i t}+\beta V_{i, t+1}^{\nu}\right)_{i \in I}$ for all $\nu$. By DU-Continuity assumed for $\succsim_{\mathbf{u}^{t-1}}$, we have $\left(u_{i t}+\beta U_{i, t+1}\right)_{i \in I} \succsim_{\mathbf{u}^{t-1}}^{*}\left(u_{i t}+\beta V_{i, t+1}\right)_{i \in I}$. By Dynamic Consistency, $U_{t+1} \succsim_{\mathbf{u}^{t-1}, u_{t}}^{*} V_{t+1}$. DU-Inequality Aversion: Let $U_{t+1} \sim_{\mathbf{u}^{t-1}, u_{t}}^{*} V_{t+1}$. By Dynamic Consistency, we have $\left(u_{i t}+\right.$ $\left.\beta U_{i, t+1}\right)_{i \in I} \sim_{\mathbf{u}^{t-1}}^{*}\left(u_{i t}+\beta V_{i, t+1}\right)_{i \in I}$. By DU-Inequality Aversion assumed for $\succsim_{\mathbf{u}^{t-1}}$, we have $\left(u_{i t}+\beta\left(c U_{i, t+1}+(1-c) V_{i, t+1}\right)\right)_{i \in I} \succsim_{\mathbf{u}^{t-1}}^{*}\left(u_{i t}+\beta U_{i, t+1}\right)_{i \in I}$. By Dynamic Consistency, $c U_{t+1}+(1-c) V_{t+1} \succsim_{\mathbf{u}^{t-1}, u_{t}}^{*} U_{t+1}$.

DU-Shift Covariance: Let $U_{t+1} \succsim_{\mathbf{u}^{t-1}, u_{t}}^{*} V_{t+1}$. By Dynamic Consistency this holds if and only if $\left(u_{i t}+\beta U_{i t}\right)_{i \in I} \succsim_{\mathbf{u}^{t-1}}^{*}\left(u_{i t}+\beta V_{i t}\right)_{i \in I}$. By DU-Shift Covariance assumed for $\succsim_{\mathbf{u}^{t-1}}$, this holds if and only if $\left(u_{i t}+\beta\left(U_{i t}+c\right)\right)_{i \in I} \succsim_{\mathbf{u}^{t-1}}^{*}\left(u_{i t}+\beta\left(V_{i t}+c\right)\right)_{i \in I}$. By Dynamic Consistency, this holds if and only if $U_{t+1}+c \mathbf{1} \succsim_{\mathbf{u}^{t-1}, u_{t}}^{*} V_{t+1}+c \mathbf{1}$. 
Theorem 4 For each fixed time $t$ and history $\mathbf{u}^{t-1}$, the social welfare ordering $\succsim_{\mathbf{u}^{t-1}}$ satisfies Order, Separability, DU-Pareto, DU-Continuity, DU-Inequality Aversion and DUShift Covariance if and only if either of the following two cases holds:

(i) there exists $\lambda\left(\mathbf{u}^{t-1}\right)>0$ and a vector $a\left(\mathbf{u}^{t-1}\right) \in$ int $\Delta(I)$ such that $\succsim_{\mathbf{u}^{t-1}}$ is represented in the form

$$
\Phi\left(\mathbf{u}_{t} \mid \mathbf{u}^{t-1}\right)=-\sum_{i \in I} a_{i}\left(\mathbf{u}^{t-1}\right) e^{-\lambda\left(\mathbf{u}^{t-1}\right) \sum_{\tau=t}^{\infty} \beta^{\tau-t} u_{i \tau}}
$$

We call this class of orderings and representations exponential class.

(ii) there exists a vector $a\left(\mathbf{u}^{t-1}\right) \in \operatorname{int} \Delta(I)$ such that $\succsim_{\mathbf{u}^{t-1}}$ is represented in the form

$$
\Phi\left(\mathbf{u}_{t} \mid \mathbf{u}^{t-1}\right)=\sum_{i \in I} a_{i}\left(\mathbf{u}^{t-1}\right) \sum_{\tau=t}^{\infty} \beta^{\tau-t} u_{i \tau}
$$

We call this class of orderings and representations additive class.

Moreover, for each $t$ and $\mathbf{u}^{t-1}$, in case (i) $a\left(\mathbf{u}^{t-1}\right)$ and $\lambda\left(\mathbf{u}^{t-1}\right)$ are unique and in case (ii) $a\left(\mathbf{u}^{t-1}\right)$ is unique.

Proof. It immediately follows from Theorem 6, a more general result.

Notice that for the exponential class we have

$$
\begin{aligned}
\Phi\left(\mathbf{u}_{0} \mid \emptyset\right) & =-\sum_{i \in I} a_{i}(\emptyset) e^{-\lambda(\emptyset) \sum_{\tau=0}^{\infty} \beta^{\tau} u_{i \tau}} \\
& =-\sum_{i \in I} a_{i}(\emptyset) e^{-\lambda(\emptyset) \sum_{\tau=0}^{t-1} \beta^{\tau} u_{i \tau}} e^{-\lambda(\emptyset) \beta^{t} \sum_{\tau=t}^{\infty} \beta^{\tau-t} u_{i \tau}},
\end{aligned}
$$

which under Dynamic Consistency yields the same ranking over $\mathbf{u}_{t}$ as $\Phi\left(\mathbf{u}_{t} \mid \mathbf{u}^{t-1}\right)=-\sum_{i \in I} a_{i}\left(\mathbf{u}^{t-1}\right) e^{-\lambda\left(\mathbf{u}^{t-1}\right) \sum_{\tau=t}^{\infty} \beta^{\tau-t} u_{i \tau}}$ does. $^{6}$

For the additive class, we have

$$
\begin{aligned}
\Phi\left(\mathbf{u}_{0} \mid \emptyset\right) & =\sum_{i \in I} a_{i}(\emptyset) \sum_{\tau=0}^{\infty} \beta^{\tau} u_{i \tau} \\
& =\sum_{i \in I} a_{i}(\emptyset) \sum_{\tau=0}^{t-1} \beta^{\tau} u_{i \tau}+\beta^{t} \sum_{i \in I} a_{i}(\emptyset) \sum_{\tau=t}^{\infty} \beta^{\tau-t} u_{i \tau}
\end{aligned}
$$

which under Dynamic Consistency yields the same ranking over $\mathbf{u}_{t}$ as $\Phi\left(\mathbf{u}_{t} \mid \mathbf{u}^{t-1}\right)=\sum_{i \in I} a_{i}\left(\mathbf{u}^{t-1}\right) \sum_{\tau=t}^{\infty} \beta^{\tau-t} u_{i \tau}$ does.

Therefore, after normalization, we obtain the following updating rule.

\footnotetext{
${ }^{6}$ Obviously it is impossible to switch from the exponential class to the additive class through updating, and vice versa.
} 
Theorem 5 The process of social welfare orderings $\left\{\succsim_{\mathbf{u}^{t-1}}\right\}$ satisfies Order, Separability, DU-Pareto, DU-Continuity, DU-Inequality Aversion and DU-Shift Covariance at $\emptyset$ and Dynamic Consistency if and only if either

(i) $\left\{\succsim_{\mathbf{u}^{t-1}}\right\}$ falls in the exponential class and $\left\{a\left(\mathbf{u}^{t}\right)\right\}$ and $\left\{\lambda\left(\mathbf{u}^{t}\right)\right\}$ follow the updating rule

$$
\begin{aligned}
a_{i}\left(\mathbf{u}^{t-1}\right) & =\frac{a_{i}(\emptyset) e^{-\lambda(\emptyset) \sum_{\tau=0}^{t-1} \beta^{\tau} u_{i \tau}}}{\sum_{j \in I} a_{j}(\emptyset) e^{-\lambda(\emptyset) \sum_{\tau=0}^{t-1} \beta^{\tau} u_{j \tau}}} \\
\lambda\left(\mathbf{u}^{t-1}\right) & =\lambda(\emptyset) \beta^{t}
\end{aligned}
$$

for all $\mathbf{u}^{t-1}$, or

(ii) $\left\{\succsim_{\mathbf{u}^{t-1}}\right\}$ falls in the additive class and $\left(a_{i}\left(\mathbf{u}^{t}\right)\right)_{i \in I}$ follows

$$
a_{i}\left(\mathbf{u}^{t-1}\right)=a_{i}(\emptyset)
$$

for all $\mathbf{u}^{t-1}$.

The theorem says that if the social ranking has equity concern it must incorporate past utilities into account in updating welfare weights as described by formula (1), while the equity concern must 'decease' over time according to the exponential order as described by formula (2). There, higher (lower) past utilities must be 'compensated' in the way that they decrease (increase) welfare weights in the exponential manner. It also says that when the social ranking has no equity concern welfare weights must be constant over time as in formula (3), and it does not take past utilities into account.

This implies that when we require that social ranking should ignore past the only possibility is additive aggregation with weights being constant over time.

History Independence: for all $t$ and for all $\mathbf{u}^{t-1}$ and $\widetilde{\mathbf{u}}^{t-1}, \succsim_{\mathbf{u}^{t-1}}=\succsim_{\widetilde{\mathbf{u}}^{t-1}}$.

Corollary 2 Suppose that the process of social welfare orderings $\left\{\succsim_{\mathbf{u}^{t-1}}\right\}$ satisfying Dynamic Consistency falls in the class as characterized in Theorem 4 and 5 . Then it satisfies History Independence if and only if it falls in the additive class with $a\left(\mathbf{u}^{t-1}\right)=a$ for all $\mathbf{u}^{t-1}$. 


\subsection{Consistent updating under heterogeneous discounting}

If we attempt to extend the previous argument on aggregating discounted utility to the case of heterogeneous discounting described by $\left(\beta_{i}\right)_{i \in I}$, we have

$$
\begin{aligned}
\Phi\left(\mathbf{u}_{0} \mid \emptyset\right) & =-\sum_{i \in I} a_{i}(\emptyset) e^{-\lambda(\emptyset) \sum_{\tau=0}^{\infty} \beta_{i}^{\tau} u_{i \tau}} \\
& =-\sum_{i \in I} a_{i}(\emptyset) e^{-\lambda(\emptyset) \sum_{\tau=0}^{t-1} \beta_{i}^{\tau} u_{i \tau}} e^{-\lambda(\emptyset) \beta_{i}^{t} \sum_{\tau=t}^{\infty} \beta_{i}^{\tau-t} u_{i \tau}}
\end{aligned}
$$

which in general cannot represent the same ranking over $\mathbf{u}_{t}$ as $\Phi\left(\mathbf{u}_{t} \mid \mathbf{u}^{t-1}\right)=-\sum_{i \in I} a_{i}\left(\mathbf{u}^{t-1}\right) e^{-\lambda\left(\mathbf{u}^{t-1}\right) \sum_{\tau=t}^{\infty} \beta^{\tau-t} u_{i \tau}}$ does, because the term $\lambda(\emptyset) \beta_{i}^{t}$ is not identical across individuals.

Therefore, the exponential class is not closed under updating under heterogeneous discounting, and we have the following result.

Proposition 2 Consider the class of social welfare functions as obtained in Theorem 4. Under heterogeneous discounting, it satisfies Dynamic Consistency holds if and only if the social welfare function is limited to the additive class with $a\left(\mathbf{u}^{t-1}\right)=a(\emptyset)$ for all $\mathbf{u}^{t-1}$.

The reason is that DU-Shift Covariance is not in general preserved under consistent updating when discounting is heterogrnous. However, a weaker version of DU-Shift Covariance is shown to be preserved under consistent updating.

DU-General Shift Covariance: there exists $W_{\mathbf{u}^{t-1}} \in \mathbb{R}_{++}^{I}$ such that for all $U_{t}, V_{t} \in \mathbb{R}^{I}$ and $c \in \mathbb{R}, U_{t} \succsim_{\mathbf{u}^{t-1}}^{*} V_{t}$ implies $U_{t}+c W_{\mathbf{u}^{t-1}} \succsim_{\mathbf{u}^{t-1}}^{*} V_{t}+c W_{\mathbf{u}^{t-1}}$.

Proposition 3 Under heterogeneous discounting, Order, Separability, DU-Pareto, DUContinuity, DU-Inequality Aversion and DU-General Shift Covariance are preserved under consistent updating.

Proof. We only prove that DU-General Shift Covariance is preserved, since the rest is similar to the case of homogeneous discounting.

Let $U_{t+1} \succsim_{\mathbf{u}^{t-1}, u_{t}}^{*} V_{t+1}$. By Dynamic Consistency this holds if and only if $\left(u_{i t}+\beta_{i} U_{i t}\right)_{i \in I} \succsim_{\mathbf{u}^{t-1}}^{*}$ $\left(u_{i t}+\beta_{i} V_{i t}\right)_{i \in I}$. By DU-General Shift Covariance assumed for $\succsim_{\mathbf{u}^{t-1}}$, for some $W_{\mathbf{u}^{t-1}}$ this holds if and only if $\left(u_{i t}+\beta_{i}\left(U_{i t}+c W_{i, \mathbf{u}^{t-1}} / \beta_{i}\right)_{i \in I} \succsim_{\mathbf{u}^{t-1}}^{*}\left(u_{i t}+\beta_{i}\left(V_{i t}+c W_{i, \mathbf{u}^{t-1}} / \beta_{i}\right)\right)_{i \in I}\right.$. By Dynamic Consistency, this holds if and only if $U_{t+1}+c W_{\mathbf{u}^{t-1}, u_{t}} \succsim_{\mathbf{u}^{t-1}, u_{t}}^{*} V_{t+1}+c W_{\mathbf{u}^{t-1}, u_{t}}$, where $W_{\mathbf{u}^{t-1}, u_{t}}=\left(W_{i, \mathbf{u}^{t-1}, u_{t}} / \beta_{i}\right)_{i \in I}$. 
Theorem 6 For each fixed time $t$ and history $\mathbf{u}^{t-1}$, the social welfare ordering $\succsim_{\mathbf{u}^{t-1}}$ satisfies Order, Separability, DU-Pareto, DU-Continuity, DU-Inequality Aversion and DUGeneral Shift Covariance if and only if either of the following two cases holds:

(i) there exists a vector $\lambda\left(\mathbf{u}^{t-1}\right) \in \mathbb{R}_{++}^{I}$ and a vector $a\left(\mathbf{u}^{t-1}\right) \in \operatorname{int} \Delta(I)$ such that $\succsim_{\mathbf{u}^{t-1}}$ is represented in the form

$$
\Phi\left(\mathbf{u}_{t} \mid \mathbf{u}^{t-1}\right)=-\sum_{i \in I} a_{i}\left(\mathbf{u}^{t-1}\right) e^{-\lambda_{i}\left(\mathbf{u}^{t-1}\right) \sum_{\tau=t}^{\infty} \beta^{\tau-t} u_{i \tau}}
$$

We call this class of orderings and representations generalized exponential class.

(ii) there exists a vector $a\left(\mathbf{u}^{t-1}\right) \in \operatorname{int} \Delta(I)$ such that $\succsim_{\mathbf{u}^{t-1}}$ is represented in the form

$$
\Phi\left(\mathbf{u}_{t} \mid \mathbf{u}^{t-1}\right)=-\sum_{i \in I} a_{i}\left(\mathbf{u}^{t-1}\right) \sum_{\tau=t}^{\infty} \beta^{\tau-t} u_{i \tau}
$$

We call this class of orderings and representations additive class.

Moreover, for each $t$ and $\mathbf{u}^{t-1}$, in case (i) $a\left(\mathbf{u}^{t-1}\right)$ and $\lambda\left(\mathbf{u}^{t-1}\right)$ are unique and in case (ii) $a\left(\mathbf{u}^{t-1}\right)$ is unique.

Proof. It follows from the fact that $\succsim_{\mathbf{u}^{t-1}}^{*}$ satisfies all the conditions in Lemma 2.

Now for the generalized exponential class we have

$$
\begin{aligned}
\Phi\left(\mathbf{u}_{0} \mid \emptyset\right) & =-\sum_{i \in I} a_{i}(\emptyset) e^{-\lambda_{i}(\emptyset) \sum_{\tau=0}^{\infty} \beta_{i}^{\tau} u_{i \tau}} \\
& =-\sum_{i \in I} a_{i}(\emptyset) e^{-\lambda_{i}(\emptyset) \sum_{\tau=0}^{t-1} \beta_{i}^{\tau} u_{i \tau}} e^{-\lambda_{i}(\emptyset) \beta_{i}^{t} \sum_{\tau=t}^{\infty} \beta_{i}^{\tau-t} u_{i \tau}},
\end{aligned}
$$

which under Dynamic Consistency yields the same ranking over $\mathbf{u}_{t}$ as $\Phi\left(\mathbf{u}_{t} \mid \mathbf{u}^{t-1}\right)=-\sum_{i \in I} a_{i}\left(\mathbf{u}^{t-1}\right) e^{-\lambda_{i}\left(\mathbf{u}^{t-1}\right) \sum_{\tau=t}^{\infty} \beta_{i}^{\tau-t} u_{i \tau}}$ does.

For the additive class, we have

$$
\begin{aligned}
\Phi\left(\mathbf{u}_{0} \mid \emptyset\right) & =\sum_{i \in I} a_{i}(\emptyset) \sum_{\tau=0}^{\infty} \beta_{i}^{\tau} u_{i \tau} \\
& =\sum_{i \in I} a_{i}(\emptyset) \sum_{\tau=0}^{t-1} \beta_{i}^{\tau} u_{i \tau}+\sum_{i \in I} a_{i}(\emptyset) \beta_{i}^{t} \sum_{\tau=t}^{\infty} \beta^{\tau-t} u_{i \tau}
\end{aligned}
$$

which under Dynamic Consistency yields the same ranking over $\mathbf{u}_{t}$ as $\Phi\left(\mathbf{u}_{t} \mid \mathbf{u}^{t-1}\right)=\sum_{i \in I} a_{i}\left(\mathbf{u}^{t-1}\right) \sum_{\tau=t}^{\infty} \beta_{i}^{\tau-t} u_{i \tau}$ does.

Therefore, after normalization, we obtain the following updating rule. 
Theorem 7 The process of social welfare orderings $\left\{\succsim_{\mathbf{u}^{t-1}}\right\}$ satisfies Order, Separability, DU-Pareto, DU-Continuity, DU-Inequality Aversion and DU-General Shift Covariance at $\emptyset$ and Dynamic Consistency if and only if either

(i) $\left\{\succsim_{\mathbf{u}^{t-1}}\right\}$ falls in the generalized exponential class and $\left\{a\left(\mathbf{u}^{t}\right)\right\}$ and $\left\{\lambda\left(\mathbf{u}^{t}\right)\right\}$ follow the updating rule

$$
\begin{aligned}
a_{i}\left(\mathbf{u}^{t-1}\right) & =\frac{a_{i}(\emptyset) e^{-\lambda(\emptyset) \sum_{\tau=0}^{t-1} \beta_{i}^{\tau} u_{i \tau}}}{\sum_{j \in I} a_{j}(\emptyset) e^{-\lambda(\emptyset) \sum_{\tau=0}^{t-1} \beta_{j}^{\tau} u_{j \tau}}} \\
\lambda_{i}\left(\mathbf{u}^{t-1}\right) & =\lambda_{i}(\emptyset) \beta_{i}^{t}
\end{aligned}
$$

for all $\mathbf{u}^{t}$, or

(ii) $\left\{\succsim_{\mathbf{u}^{t-1}}\right\}$ falls in the additive class and $\left(a_{i}\left(\mathbf{u}^{t}\right)\right)_{i \in I}$ follows the updating rule

$$
a_{i}\left(\mathbf{u}^{t-1}\right)=\frac{a_{i}(\emptyset) \beta_{i}^{t}}{\sum_{j \in I} a_{j}(\emptyset) \beta_{j}^{t}} .
$$

for all $\mathbf{u}^{t-1}$.

The theorem says that if the social ranking has equity concern it must incorporate past utilities into account in updating welfare weights as described by formula (4), while the equity concern on each individual deceases over time according to her discount factor as in formula (5). There, higher (lower) past utilities must be 'compensated' in the way that they decrease (increase) welfare weights in the exponential manner. It also says that when the social ranking has no equity concern welfare weights evolve according to individuals' discount factors as in formula (6), which depends on time index but does not take past utilities into account.

This implies that when we require that social ranking should ignore past past utilities the only possibility is additive aggregation.

Corollary 3 Suppose that the process of social welfare orderings $\left\{\succsim_{\mathbf{u}^{t-1}}\right\}$ falls in the class as characterized in Theorem 6 and 7. Then it satisfies Dynamic Consistency and History Independence hold if and only if it falls in the additive class in which the updating of welfare weights follows

$$
a_{i}\left(\mathbf{u}^{t-1}\right)=\frac{a_{i}(\emptyset) \beta_{i}^{t}}{\sum_{j \in I} a_{j}(\emptyset) \beta_{j}^{t}}
$$

for all $\mathbf{u}^{t-1}$. 
Also, the above result implies that under heterogeneous discounting the social ranking cannot be independent of time index.

History-Time Independence: for all $t, s$ and for all $\mathbf{u}^{t-1}$ and $\widetilde{\mathbf{u}}^{s-1}, \succsim_{\mathbf{u}^{t-1}}=\succsim_{\widetilde{\mathbf{u}}^{s-1}}$.

Corollary 4 Under heterogeneous discounting, there is no process of social welfare orderings which satisfies Dynamic Consistency and History-Time Independence.

\section{Consistent updating under uncertainty}

\subsection{Consistent updating under homogeneous beliefs}

$\mathrm{n}$ this section, we demonstrate how the axioms imposed on static social welfare functions are preserved under consistent updating along with resolution of uncertainty. Here we limit attention to the case of common belief across individuals and we will extend the argument to the case of heterogeneous beliefs in a later section.

Let $\Omega$ be the set of states of the world, which is a measure space with a common prior $p$ having full support. Given a measurable subset $E \subset \Omega$ and a subset of individuals $J \subset I$, let $L^{J \times E}$ be the set of integrable functions from $E$ to $\mathbb{R}^{J}$, which is interpreted as a set of random utility profiles for group $J$ conditional on $E$.

Let $\left\{\mathcal{E}_{t}\right\}$ be the sequence of increasing partitions of $\Omega$, which describes how uncertainties are resolved over time. Given $t$ and $E_{t} \in \mathcal{E}_{t}$, let $\mathbf{u}_{-E_{t}} \in L^{I \times\left(\Omega \backslash E_{t}\right)}$ be an integrable random utility profile defined over $\Omega \backslash E_{t}$, which describes what individuals utilities would have been if $E_{t}$ had not occurred. Denote the initial point with no such foregone utilities by $\emptyset$. For simplicity we restrict attention to utility values at final states, which can easily be combine with the argument with intertemporal consumptions as presented in the previous section. Given $E_{t} \in \mathcal{E}_{t}$, let $p\left(\cdot \mid E_{t}\right)$ be its Bayesian update of $p$ conditional on $E_{t}$.

For each possible $\mathbf{u}_{-E_{t}}$, let $\succsim_{\mathbf{u}_{-E_{t}}}$ be the social welfare ordering over $L^{I \times E_{t}}$ conditional on $\mathbf{u}_{-E_{t}}$. Given $\mathbf{u}_{E_{t}}, \mathbf{v}_{E_{t}} \in L^{I \times E_{t}}$, the ranking may be written for example as $\mathbf{u}_{E_{t}} \succsim_{\mathbf{u}_{-E_{t}}} \mathbf{v}_{E_{t}}$. Let $\left\{\succsim_{\mathbf{u}_{-E_{t}}}\right\}$ be a process of such social welfare orderings.

Again, we will consider two kinds of axioms. One is properties of social welfare criteria which are desirable to be satisfied at each time and possible event, and are investigated if they are preserved under consistent updating along with time passing and realization of uncertainty. We call such axioms intra-profile axioms. The other is about relationships 
between social welfare orderings indexed with different times and possible events. We call such axioms inter-profile axioms.

Dynamic consistency condition is the inter-profile axiom we impose throughout the analysis.

Dynamic Consistency: for all $t, E_{t}$ and $\mathbf{u}_{-E_{t}}$, for all $E_{t+1} \in \mathcal{E}_{t+1} \cap E_{t}$ and $\mathbf{u}_{E_{t} \backslash E_{t+1}} \in$ $L^{I \times\left(E_{t} \backslash E_{t+1}\right)}$, and for all $\mathbf{u}_{E_{t+1}}, \mathbf{v}_{E_{t+1}} \in L^{I \times E_{t+1}}$,

$$
\left(\mathbf{u}_{E_{t+1}}, \mathbf{u}_{E_{t} \backslash E_{t+1}}\right) \succsim_{\mathbf{u}_{-E_{t}}}\left(\mathbf{v}_{E_{t+1}}, \mathbf{u}_{E_{t} \backslash E_{t+1}}\right) \Longleftrightarrow \mathbf{u}_{E_{t+1}} \succsim_{\mathbf{u}_{-E_{t}}, \mathbf{u}_{E_{t} \backslash E_{t+1}}} \mathbf{v}_{E_{t+1}}
$$

We define the condition that an intra-profile axiom is preserved under consistent updating across times.

Definition 4 An intra-profile axiom is said to be preserved under consistent updating if for any process of social welfare orderings $\left\{\succsim_{\mathbf{u}_{-E_{t}}}\right\}$ satisfying Dynamic Consistency, for all $t, E_{t}$ and $\mathbf{u}_{-E_{t}}$, for all $E_{t+1} \in \mathcal{E}_{t+1} \cap E_{t}$ and $\mathbf{u}_{E_{t} \backslash E_{t+1}} \in L^{I \times\left(E_{t} \backslash E_{t+1}\right)}$, if it being stated on

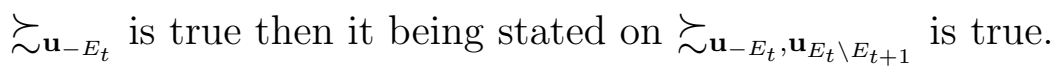

Example 4 The following intra-profile axioms are not preserved under consistent updating.

Anonymity at given $t, E_{t}$ and $\mathbf{u}_{-E_{t}}$ : for all $\mathbf{u}_{E_{t}}, \mathbf{v}_{E_{t}} \in L^{I \times E_{t}}$, and any permutation $\pi$ over $I$,

$$
\mathbf{u}_{E_{t}} \succsim \mathbf{u}_{-E_{t}} \mathbf{v}_{E_{t}} \Longleftrightarrow \mathbf{u}_{E_{t}}^{\pi} \succsim \mathbf{u}_{-E_{t}} \mathbf{v}_{E_{t}}^{\pi} \text {. }
$$

Homogeneity at given $t, E_{t}$ and $\mathbf{u}_{-E_{t}}$ : for all $\mathbf{u}_{E_{t}}, \mathbf{v}_{E_{t}} \in L^{I \times E_{t}}$, and for all non-negative number $c$,

$$
\mathbf{u}_{E_{t}} \succsim \mathbf{u}_{-E_{t}} \mathbf{v}_{E_{t}} \Longleftrightarrow c \mathbf{u}_{E_{t}} \succsim \mathbf{u}_{-E_{t}} c \mathbf{v}_{E_{t}}
$$

To see that these axioms are not preserved under updating, consider the social welfare function at $\mathbf{u}_{-E_{t}}$ in the form

$$
\Phi\left(\mathbf{u}_{E_{t}} \mid \mathbf{u}_{-E_{t}}\right)=\min _{i \in I} \int_{E_{t}} u_{i}(s) p\left(d s \mid E_{t}\right)
$$

This obviously satisfies Anonymity and Homogeneity at $\mathbf{u}_{-E_{t}}$. However, its consistent updating $\Phi\left(\mathbf{u}_{E_{t+1}} \mid \mathbf{u}_{-E_{t}}, \mathbf{u}_{E_{t} \backslash E_{t+1}}\right)$ at $\left(\mathbf{u}_{-E_{t}}, \mathbf{u}_{E_{t} \backslash E_{t+1}}\right)$ must be ordinally equivalent to the function

$$
\min _{i \in I}\left(p\left(E_{t} \backslash E_{t+1} \mid E_{t}\right) \int_{E_{t+1}} u_{i}(s) p\left(d s \mid E_{t} \backslash E_{t+1}\right)+p\left(E_{t+1} \mid E_{t}\right) \int_{E_{t+1}} u_{i}(s) p\left(d s \mid E_{t+1}\right)\right),
$$


which does not satisfy Anonymity or Homogeneity as the ranking over $\left(\mathbf{u}_{-E_{t}}, \mathbf{u}_{E_{t} \backslash E_{t+1}}\right)$.

Again, the reason is rather easier to see at a conceptual level. Note that these axioms are about renaming and rescaling still possible utilities. Anonymity says that identity of individuals does not matter in evaluating still possible utilities. However, in the presence of events which did not occur but might have delivered different utilities to different individuals, identity of the individuals is indispensable in consistent updating. Homogeneity says that changing the common scale of possible individual utilities does not matter. However, changing the scale of still possible utilities changes the impact of utilities which turned not to occur, and it in general results in violating dynamic consistency.

To introduce intra-profile axioms, fix an arbitrarily time $t, E_{t}$ and $\mathbf{u}_{-E_{t}}$. The first one is self-explanatory.

Order: $\succsim_{\mathbf{u}_{-E_{t}}}$ is complete and transitive ordering over $L^{I \times E_{t}}$.

Second we consider a group-separability axiom.

Separability: for all $J \subset I$, for all $\mathbf{u}_{J, E_{t}}, \mathbf{v}_{J, E_{t}} \in L^{J \times E_{t}}$ and for all $\mathbf{u}_{-J, E_{t}}, \mathbf{v}_{-J, E_{t}} \in$ $L^{(I \backslash J) \times E_{t}},\left(\mathbf{u}_{J, E_{t}}, \mathbf{u}_{-J, E_{t}}\right) \succsim_{\mathbf{u}_{-E_{t}}}\left(\mathbf{v}_{J, E_{t}}, \mathbf{u}_{-J, E_{t}}\right)$ holds if and only if $\left(\mathbf{u}_{J, E_{t}}, \mathbf{v}_{-J, E_{t}}\right) \succsim_{\mathbf{u}_{-E_{t}}}$ $\left(\mathbf{v}_{J, E_{t}}, \mathbf{v}_{-J, E_{t}}\right)$.

Next we impose that the social ranking over utility streams depends only on individuals' expected utility evaluations. This says that each individual is taken to be responsible for summarizing her own utilities at different states, and the social ranking concerns only such summaries. One may see the non-triviality of this condition by thinking for example of treating inequalities across both individuals and states in tandem, which is excluded by the axiom (see the discussions in Ben-Porath, Gilboa and Schmeidler [1]).

EU-Pareto: for all $\mathbf{u}_{E_{t}}, \mathbf{v}_{E_{t}} \in L^{I \times E_{t}}, \int_{E_{t}} u_{i}(s) p\left(d s \mid E_{t}\right) \geq \int_{E_{t}} v_{i}(s) p\left(d s \mid E_{t}\right)$ for all $i \in I$ implies $\mathbf{u}_{E_{t}} \succsim \mathbf{u}_{-E_{t}} \mathbf{v}_{E_{t}}$, and the conclusion is strict additionally if $\int_{E_{t}} u_{i}(s) p\left(d s \mid E_{t}\right)>$ $\int_{E_{t}} v_{i}(s) p\left(d s \mid E_{t}\right)$ for some $i \in I$.

Under EU-Pareto, one can define a social ranking induced over expected utilities.

Definition 5 Given $t, E_{t}$ and $\mathbf{u}_{-E_{t}}$, the EU-welfare ordering $\succsim_{\mathbf{u}_{-E_{t}}}$ over $\mathbb{R}^{I}$ induced by $\succsim_{\mathbf{u}_{-E_{t}}}$ is defined by

$$
U_{E_{t}} \succsim_{\mathbf{u}_{-E_{t}}}^{*} V_{E_{t}} \Longleftrightarrow \mathbf{u}_{E_{t}} \succsim \mathbf{u}_{-E_{t}} \mathbf{v}_{E_{t}}
$$


for $\mathbf{u}_{E_{t}}, \mathbf{v}_{E_{t}} \in \mathbb{R}^{I \times E_{t}}$ with $U_{E_{t}}=\int_{E_{t}} u_{i}(s) p\left(d s \mid E_{t}\right)$ and $V_{E_{t}}=\int_{E_{t}} v_{i}(s) p\left(d s \mid E_{t}\right)$ respectively. The EU-Pareto axiom ensures that this is well-defined.

We impose the following three axioms on the induced ranking. Although one can write them down directly in terms of the original ranking, this helps simplifying the exposition.

EU-Continuity: $\succsim_{\mathbf{u}_{-E_{t}}}^{*}$ is a closed subset of $\mathbb{R}^{I} \times \mathbb{R}^{I}$.

EU-Inequality Aversion: for all $U_{E_{t}}, V_{E_{t}} \in \mathbb{R}^{I}$ and $c \in[0,1], U_{E_{t}} \sim_{\mathbf{u}_{-E_{t}}}^{*} V_{E_{t}}$ implies $c U_{E_{t}}+(1-c) V_{E_{t}} \succsim_{\mathbf{u}_{-E_{t}}}^{*} V_{E_{t}}$.

EU-Shift Covariance: for all $U_{E_{t}}, V_{E_{t}} \in \mathbb{R}^{I}$ and $c \in \mathbb{R}, U_{E_{t}} \succsim_{\mathbf{u}_{-E_{t}}}^{*} V_{E_{t}}$ implies $U_{E_{t}}+$ $c \mathbf{1} \succsim_{\mathbf{u}_{-E_{t}}}^{*} V_{E_{t}}+c \mathbf{1}$.

Proposition 4 Order, Separability, EU-Pareto, EU-Continuity, EU-Inequality Aversion and EU-Shift Covariance are preserved under consistent updating.

\section{Proof.}

Order: Obvious.

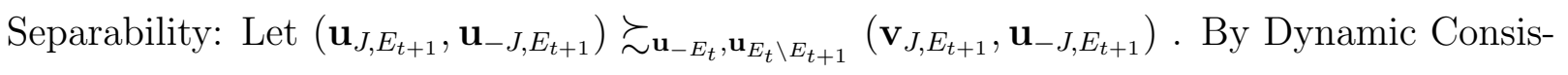
tency, this holds if and only if

$\left(\left(\mathbf{u}_{J, E_{t} \backslash E_{t+1}}, \mathbf{u}_{J, E_{t+1}}\right),\left(\mathbf{u}_{-J, E_{t} \backslash E_{t+1}}, \mathbf{u}_{-J, E_{t+1}}\right)\right) \succsim_{\mathbf{u}_{-E_{t}}}\left(\left(\mathbf{u}_{J, E_{t} \backslash E_{t+1}}, \mathbf{v}_{J, E_{t+1}}\right),\left(\mathbf{u}_{-J, E_{t} \backslash E_{t+1}}, \mathbf{u}_{-J, E_{t+1}}\right)\right)$.

By Separability assumed for $\succsim_{\mathbf{u}_{-E_{t}}}$, this holds if and only if

$\left(\left(\mathbf{u}_{J, E_{t} \backslash E_{t+1}}, \mathbf{u}_{J, E_{t+1}}\right),\left(\mathbf{u}_{-J, E_{t} \backslash E_{t+1}}, \mathbf{v}_{-J, E_{t+1}}\right)\right) \succsim_{\mathbf{u}_{-E_{t}}}\left(\left(\mathbf{u}_{J, E_{t} \backslash E_{t+1}}, \mathbf{v}_{J, E_{t+1}}\right),\left(\mathbf{u}_{-J, E_{t} \backslash E_{t+1}}, \mathbf{v}_{-J, E_{t+1}}\right)\right)$.

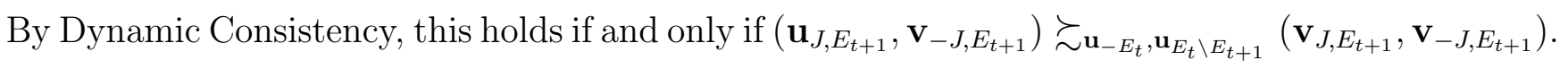
EU-Pareto: Let $\int_{E_{t+1}} u_{i}(s) p\left(d s \mid E_{t+1}\right) \geq \int_{E_{t+1}} v_{i}(s) p\left(d s \mid E_{t+1}\right)$ for all $i \in I$ for all $i \in I$. Then we have

$$
\begin{aligned}
& p\left(E_{t} \backslash E_{t+1} \mid E_{t}\right) \int_{E_{t+1}} u_{i}(s) p\left(d s \mid E_{t} \backslash E_{t+1}\right)+p\left(E_{t+1} \mid E_{t}\right) \int_{E_{t+1}} u_{i}(s) p\left(d s \mid E_{t+1}\right) \\
\geq & p\left(E_{t} \backslash E_{t+1} \mid E_{t}\right) \int_{E_{t+1}} u_{i}(s) p\left(d s \mid E_{t} \backslash E_{t+1}\right)+p\left(E_{t+1} \mid E_{t}\right) \int_{E_{t+1}} v_{i}(s) p\left(d s \mid E_{t+1}\right)
\end{aligned}
$$

for all $i \in I$. By EU-Pareto assumed for $\succsim_{\mathbf{u}_{-E_{t}}}$, we have $\left(\mathbf{u}_{E_{t+1}}, \mathbf{u}_{E_{t} \backslash E_{t+1}}\right) \succsim_{\mathbf{u}_{-E_{t}}}\left(\mathbf{v}_{E_{t+1}}, \mathbf{u}_{E_{t} \backslash E_{t+1}}\right)$.

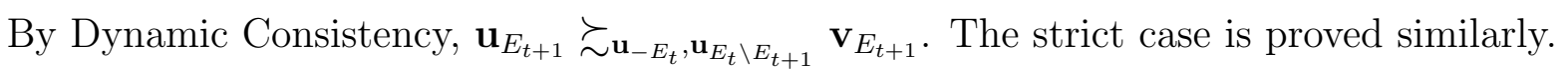


EU-Continuity: Suppose $U_{E_{t+1}}^{\nu} \succsim_{\mathbf{u}_{-E_{t}}, \mathbf{u}_{E_{t} \backslash E_{t+1}}} V_{E_{t+1}}^{\nu}$ for all $\nu$. By Dynamic Consistency, we have

$$
\begin{aligned}
& \left(p\left(E_{t} \backslash E_{t+1} \mid E_{t}\right) \int_{E_{t+1}} u_{i}(s) p\left(d s \mid E_{t} \backslash E_{t+1}\right)+p\left(E_{t+1} \mid E_{t}\right) U_{i, E_{t+1}}^{\nu}\right)_{i \in I} \\
\succsim^{*} \mathbf{u}_{-E_{t}} & \left(p\left(E_{t} \backslash E_{t+1} \mid E_{t}\right) \int_{E_{t+1}} u_{i}(s) p\left(d s \mid E_{t} \backslash E_{t+1}\right)+p\left(E_{t+1} \mid E_{t}\right) V_{i, E_{t+1}}^{\nu}\right)_{i \in I}
\end{aligned}
$$

for all $\nu$. By EU-Continuity assumed for $\succsim_{\mathbf{u}_{-E_{t}}}$, we have

$$
\begin{aligned}
& \left(p\left(E_{t} \backslash E_{t+1} \mid E_{t}\right) \int_{E_{t+1}} u_{i}(s) p\left(d s \mid E_{t} \backslash E_{t+1}\right)+p\left(E_{t+1} \mid E_{t}\right) U_{i, E_{t+1}}\right)_{i \in I} \\
\succsim_{\mathbf{u}_{-E_{t}}}^{*} & \left(p\left(E_{t} \backslash E_{t+1} \mid E_{t}\right) \int_{E_{t+1}} u_{i}(s) p\left(d s \mid E_{t} \backslash E_{t+1}\right)+p\left(E_{t+1} \mid E_{t}\right) V_{i, E_{t+1}}\right)_{i \in I} .
\end{aligned}
$$

By Dynamic Consistency, $U_{E_{t+1}} \succsim_{\mathbf{u}_{-E_{t}}, \mathbf{u}_{E_{t} \backslash E_{t+1}}} V_{E_{t+1}}$.

EU-Inequality Aversion: Let $U_{E_{t+1}} \sim_{\mathbf{u}_{-E_{t}}, \mathbf{u}_{E_{t} \backslash E_{t+1}}}^{*} V_{E_{t+1}}$. By Dynamic Consistency, we have

$$
\begin{aligned}
& \left(p\left(E_{t} \backslash E_{t+1} \mid E_{t}\right) \int_{E_{t+1}} u_{i}(s) p\left(d s \mid E_{t} \backslash E_{t+1}\right)+p\left(E_{t+1} \mid E_{t}\right) U_{i, E_{t+1}}\right)_{i \in I} \\
\sim_{\mathbf{u}_{-E_{t}}}^{*} & \left(p\left(E_{t} \backslash E_{t+1} \mid E_{t}\right) \int_{E_{t+1}} u_{i}(s) p\left(d s \mid E_{t} \backslash E_{t+1}\right)+p\left(E_{t+1} \mid E_{t}\right) V_{i, E_{t+1}}\right)_{i \in I} .
\end{aligned}
$$

By EU-Inequality Aversion assumed for $\succsim_{\mathbf{u}_{-E_{t}}}$, we have

$$
\begin{aligned}
& \left(p\left(E_{t} \backslash E_{t+1} \mid E_{t}\right) \int_{E_{t+1}} u_{i}(s) p\left(d s \mid E_{t} \backslash E_{t+1}\right)+p\left(E_{t+1} \mid E_{t}\right)\left(c U_{i, E_{t+1}}+(1-c) V_{i, E_{t+1}}\right)\right)_{i \in I} \\
\succsim_{\mathbf{u}_{-E_{t}}}^{*} & \left(p\left(E_{t} \backslash E_{t+1} \mid E_{t}\right) \int_{E_{t+1}} u_{i}(s) p\left(d s \mid E_{t} \backslash E_{t+1}\right)+p\left(E_{t+1} \mid E_{t}\right) V_{i, E_{t+1}}\right)_{i \in I} .
\end{aligned}
$$

By Dynamic Consistency, $c U_{E_{t+1}}+(1-c) V_{E_{t+1}} \succsim_{\mathbf{u}_{-E_{t}}, \mathbf{u}_{E_{t} \backslash E_{t+1}}}^{*} U_{E_{t+1}}$.

EU-Shift Covariance: Let $U_{E_{t+1}} \succsim_{\mathbf{u}_{-E_{t}}, \mathbf{u}_{E_{t} \backslash E_{t+1}}} V_{E_{t+1}}$. By Dynamic Consistency this holds if and only if

$$
\begin{aligned}
& \left(p\left(E_{t} \backslash E_{t+1} \mid E_{t}\right) \int_{E_{t+1}} u_{i}(s) p\left(d s \mid E_{t} \backslash E_{t+1}\right)+p\left(E_{t+1} \mid E_{t}\right) U_{i, E_{t+1}}\right)_{i \in I} \\
\succsim_{\mathbf{u}_{-E_{t}}}^{*} & \left(p\left(E_{t} \backslash E_{t+1} \mid E_{t}\right) \int_{E_{t+1}} u_{i}(s) p\left(d s \mid E_{t} \backslash E_{t+1}\right)+p\left(E_{t+1} \mid E_{t}\right) V_{i, E_{t+1}}\right)_{i \in I} .
\end{aligned}
$$

By EU-Shift Covariance assumed for $\succsim_{\mathbf{u}_{-E_{t}}}$, this holds if and only if

$$
\begin{aligned}
& \left(p\left(E_{t} \backslash E_{t+1} \mid E_{t}\right) \int_{E_{t+1}} u_{i}(s) p\left(d s \mid E_{t} \backslash E_{t+1}\right)+p\left(E_{t+1} \mid E_{t}\right)\left(U_{i, E_{t+1}}+c\right)\right)_{i \in I} \\
\succsim_{\mathbf{u}_{-E_{t}}}^{*} & \left(p\left(E_{t} \backslash E_{t+1} \mid E_{t}\right) \int_{E_{t+1}} u_{i}(s) p\left(d s \mid E_{t} \backslash E_{t+1}\right)+p\left(E_{t+1} \mid E_{t}\right)\left(V_{i, E_{t+1}}+c\right)\right)_{i \in I} .
\end{aligned}
$$


By Dynamic Consistency, this holds if and only if $U_{E_{t+1}}+c \mathbf{1} \succsim_{\mathbf{u}_{-E_{t}}, \mathbf{u}_{E_{t} \backslash E_{t+1}}} V_{E_{t+1}}+c \mathbf{1}$.

Theorem 8 For each fixed $t, E_{t}$ and $\mathbf{u}_{-E_{t}}$, the social welfare ordering $\succsim_{\mathbf{u}_{-E_{t}}}$ satisfies Order, Separability, EU-Pareto, EU-Continuity, EU-Inequality Aversion and EU-Shift Covariance if and only if either of the following two cases holds:

(i) there exists $\lambda\left(\mathbf{u}_{-E_{t}}\right)>0$ and a vector $a\left(\mathbf{u}_{-E_{t}}\right) \in$ int $\Delta(I)$ such that $\succsim_{\mathbf{u}_{-E_{t}}}$ is represented in the form

$$
\Phi\left(\mathbf{u}_{E_{t}} \mid \mathbf{u}_{-E_{t}}\right)=-\sum_{i \in I} a_{i}\left(\mathbf{u}_{-E_{t}}\right) e^{-\lambda\left(\mathbf{u}_{-E_{t}}\right) \int_{E_{t}} u_{i}(s) p\left(d s \mid E_{t}\right)}
$$

We call this class of orderings and representations exponential class.

(ii) there exists a vector $a\left(\mathbf{u}_{-E_{t}}\right) \in$ int $\Delta(I)$ such that $\succsim_{\mathbf{u}_{-E_{t}}}$ is represented in the form

$$
\Phi\left(\mathbf{u}_{E_{t}} \mid \mathbf{u}_{-E_{t}}\right)=\sum_{i \in I} a_{i}\left(\mathbf{u}_{-E_{t}}\right) \int_{E_{t}} u_{i}(s) p\left(d s \mid E_{t}\right)
$$

We call this class of orderings and representations additive class.

Moreover, for each $t$ and $\mathbf{u}_{-E_{t}}$, in case (i) $a\left(\mathbf{u}_{-E_{t}}\right)$ and $\lambda\left(\mathbf{u}_{-E_{t}}\right)$ are unique and in case (ii) $a\left(\mathbf{u}_{-E_{t}}\right)$ is unique.

Proof. It immediately follows from Theorem 10, a more general result.

Notice that for the exponential class we have

$$
\begin{aligned}
\Phi\left(\mathbf{u}_{\Omega} \mid \emptyset\right) & =-\sum_{i \in I} a_{i}(\emptyset) e^{-\lambda(\emptyset) \int_{\Omega} u_{i}(s) p(d s)} \\
& =-\sum_{i \in I} a_{i}(\emptyset) e^{-\lambda(\emptyset) p\left(\Omega \backslash E_{t}\right) \int_{\Omega \backslash E_{t}} u_{i}(s) p\left(d s \mid \Omega \backslash E_{t}\right)} e^{-\lambda(\emptyset) p\left(E_{t}\right) \int_{E_{t}} u_{i}(s) p\left(d s \mid E_{t}\right)},
\end{aligned}
$$

which under Dynamic Consistency yields the same ranking over $\mathbf{u}_{E_{t}}$ as $\Phi\left(\mathbf{u}_{E_{t}} \mid \mathbf{u}_{-E_{t}}\right)=$ $-\sum_{i \in I} a_{i}\left(\mathbf{u}_{-E_{t}}\right) e^{-\lambda\left(\mathbf{u}_{-E_{t}}\right) \int_{E_{t}} u_{i}(s) p\left(d s \mid E_{t}\right)}$ does.

For the additive class, we have

$$
\begin{aligned}
\Phi\left(\mathbf{u}_{\Omega} \mid \emptyset\right) & =\sum_{i \in I} a_{i}(\emptyset) \int_{\Omega} u_{i}(s) p(d s) \\
& =p\left(\Omega \backslash E_{t}\right) \sum_{i \in I} a_{i}(\emptyset) \int_{\Omega \backslash E_{t}} u_{i}(s) p\left(d s \mid \Omega \backslash E_{t}\right)+p\left(E_{t}\right) \sum_{i \in I} a_{i}(\emptyset) \int_{E_{t}} u_{i}(s) p\left(d s \mid E_{t}\right),
\end{aligned}
$$

which under Dynamic Consistency yields the same ranking over $\mathbf{u}_{E_{t}}$ as $\Phi\left(\mathbf{u}_{E_{t}} \mid \mathbf{u}_{-E_{t}}\right)=$ $\sum_{i \in I} a_{i}\left(\mathbf{u}_{-E_{t}}\right) \int_{E_{t}} u_{i}(s) p\left(d s \mid E_{t}\right)$ does.

Therefore, after normalization, we obtain the following updating rule. 
Theorem 9 The process of social welfare orderings $\left\{\succsim_{\mathbf{u}_{-E_{t}}}\right\}$ satisfies Order, Separability, EU-Pareto, EU-Continuity, EU-Inequality Aversion and EU-Shift Covariance at $\emptyset$ and Dynamic Consistency if and only if either

(i) $\left\{\succsim_{\mathbf{u}_{-E_{t}}}\right\}$ falls in the exponential class and $\left\{a\left(\mathbf{u}_{-E_{t}}\right)\right\}$ and $\left\{\lambda\left(\mathbf{u}_{-E_{t}}\right)\right\}$ follow the updating rule

$$
\begin{aligned}
a_{i}\left(\mathbf{u}_{-E_{t}}\right) & =\frac{a_{i}(\emptyset) e^{-\lambda(\emptyset) p\left(\Omega \backslash E_{t}\right) \int_{\Omega \backslash E_{t}} u_{i}(s) p\left(d s \mid \Omega \backslash E_{t}\right)}}{\sum_{j \in I} a_{j}(\emptyset) e^{-\lambda(\emptyset) p\left(\Omega \backslash E_{t}\right) \int_{\Omega \backslash E_{t}} u_{j}(s) p\left(d s \mid \Omega \backslash E_{t}\right)}} \\
\lambda\left(\mathbf{u}_{-E_{t}}\right) & =\lambda(\emptyset) p\left(E_{t}\right)
\end{aligned}
$$

for all $\mathbf{u}_{-E_{t}}$, or

(ii) $\left\{\succsim_{\mathbf{u}_{-E_{t}}}\right\}$ falls in the additive class and $\left\{a\left(\mathbf{u}_{-E_{t}}\right)\right\}$ follows

$$
a_{i}\left(\mathbf{u}_{-E_{t}}\right)=a_{i}(\emptyset)
$$

for all $\mathbf{u}_{-E_{t}}$.

The theorem says that if the social ranking has equity concern it must incorporate utilities which turned out not to occur into account in updating welfare weights as described by formula (7), while the equity concern must 'decease' probabilistically over time according to formula (8). There, higher (lower) utilities which turned out not to occur must be 'compensated' in the way that they decrease (increase) welfare weights conditional on the present event. It also says that when the social ranking has no equity concern welfare weights must be constant as in formula (9), and it does not take utilities which turned out not to occur into account.

This implies that when we require that social ranking should ignore utilities which turned out not to occur the only possibility is additive aggregation with weights being constant.

Independence of Non-occurred Consequences: for all $t$, for all $E_{t}$, for all $\mathbf{u}_{-E_{t}}$ and $\widetilde{\mathbf{u}}_{-E_{t}}$, and for all $U, V \in \mathbb{R}^{I}$,

$$
\left(U_{i} \mathbf{1}_{E_{t}}\right)_{i \in I} \succsim_{\mathbf{u}_{-E_{t}}}\left(V_{i} \mathbf{1}_{E_{t}}\right)_{i \in I} \Longleftrightarrow\left(U_{i} \mathbf{1}_{E_{t}}\right)_{i \in I} \succsim_{\widetilde{\mathbf{u}}_{-E_{t}}}\left(V_{i} \mathbf{1}_{E_{t}}\right)_{i \in I}
$$

Corollary 5 Suppose that the process of social welfare orderings $\left\{\succsim_{\mathbf{u}_{-E_{t}}}\right\}$ satisfying Dynamic Consistency falls in the exponential class as characterized in Theorem 8 and 9. Then it satisfies Independence of Non-occurred Consequences if and only if it falls in the additive class with $a\left(\mathbf{u}_{-E_{t}}\right)=a$ for all $\mathbf{u}_{-E_{t}}$. 


\subsection{Consistent updating under heterogeneous beliefs}

If we attempt to extend the previous argument on aggregating expected utility to the case of heterogeneous beliefs denoted by $\left(p_{i}\right)_{i \in I}$, for the exponential class we have

$$
\begin{aligned}
\Phi\left(\mathbf{u}_{\Omega} \mid \emptyset\right) & =-\sum_{i \in I} a_{i}(\emptyset) e^{-\lambda(\emptyset) \int_{\Omega} u_{i}(s) p_{i}(d s)} \\
& =-\sum_{i \in I} a_{i}(\emptyset) e^{-\lambda(\emptyset) p_{i}\left(\Omega \backslash E_{t}\right) \int_{\Omega \backslash E_{t}} u_{i}(s) p_{i}\left(d s \mid \Omega \backslash E_{t}\right)} e^{-\lambda(\emptyset) p_{i}\left(E_{t}\right) \int_{E_{t}} u_{i}(s) p_{i}\left(d s \mid E_{t}\right)},
\end{aligned}
$$

which in general cannot represent the same ranking over $\mathbf{u}_{E_{t}}$ as $\Phi\left(\mathbf{u}_{E_{t}} \mid \mathbf{u}_{-E_{t}}\right)=-\sum_{i \in I} a_{i}\left(\mathbf{u}_{-E_{t}}\right) e^{-\lambda\left(\mathbf{u}_{-E_{t}}\right)}$ does, because the term $\lambda(\emptyset) p_{i}\left(E_{t}\right)$ is not identical across individuals.

Therefore, the exponential class is not closed under updating under heterogeneous beliefs, and we have the following result.

Proposition 5 Consider the class of social welfare functions as obtained in Theorem 8. Under heterogeneous beliefs, it satisfies Dynamic Consistency holds if and only if the social welfare function is limited to the additive class with $a\left(\mathbf{u}_{-E_{t}}\right)=a(\emptyset)$ for all $\mathbf{u}_{-E_{t}}$.

The reason is that EU-Shift Covariance is not in general preserved under consistent updating when beliefs are heterogeneous. However, a weaker version of EU-Shift Covariance is shown to be preserved under consistent updating.

EU-General Shift Covariance: there exists $W_{\mathbf{u}_{-E_{t}}} \in \mathbb{R}_{++}^{I}$ such that for all $U_{E_{t}}, V_{E_{t}} \in$ $\mathbb{R}^{I}$ and $c \in \mathbb{R}, U_{E_{t}} \succsim_{\mathbf{u}_{-E_{t}}}^{*} V_{E_{t}}$ implies $U_{E_{t}}+c W_{\mathbf{u}_{-E_{t}}} \succsim_{\mathbf{u}_{-E_{t}}}^{*} V_{E_{t}}+c W_{\mathbf{u}_{-E_{t}}}$.

Proposition 6 Under heterogeneous beliefs, Order, Separability, EU-Pareto, EU-Continuity, EU-Inequality Aversion and EU-General Shift Covariance are preserved under consistent updating.

Proof. We only prove that EU-General Shift Covariance is preserved, since the rest is similar to the case homogeneous beliefs.

Let $U_{E_{t+1}} \succsim_{\mathbf{u}_{-E_{t}}, \mathbf{u}_{E_{t} \backslash E_{t+1}}} V_{E_{t+1}}$. By Dynamic Consistency this holds if and only if

$$
\begin{aligned}
& \left(p_{i}\left(E_{t} \backslash E_{t+1} \mid E_{t}\right) \int_{E_{t+1}} u_{i}(s) p_{i}\left(d s \mid E_{t} \backslash E_{t+1}\right)+p_{i}\left(E_{t+1} \mid E_{t}\right) U_{i, E_{t+1}}\right)_{i \in I} \\
\succsim_{\mathbf{u}_{-E_{t}}}^{*} & \left(p_{i}\left(E_{t} \backslash E_{t+1} \mid E_{t}\right) \int_{E_{t+1}} u_{i}(s) p_{i}\left(d s \mid E_{t} \backslash E_{t+1}\right)+p_{i}\left(E_{t+1} \mid E_{t}\right) V_{i, E_{t+1}}\right)_{i \in I} .
\end{aligned}
$$


By EU-General Shift Covariance assumed for ${\succsim \mathbf{u}_{-E_{t}}}_{\text {}}$, for some $W_{\mathbf{u}_{-E_{t}}}$ this holds if and only if

$$
\begin{aligned}
& \left(p_{i}\left(E_{t} \backslash E_{t+1} \mid E_{t}\right) \int_{E_{t+1}} u_{i}(s) p_{i}\left(d s \mid E_{t} \backslash E_{t+1}\right)+p_{i}\left(E_{t+1} \mid E_{t}\right)\left(U_{i, E_{t+1}}+c \frac{W_{\mathbf{u}_{-E_{t}}}}{p_{i}\left(E_{t+1} \mid E_{t}\right)}\right)\right)_{i \in I} \\
\succsim_{\mathbf{u}_{-E_{t}}}^{*} & \left(p_{i}\left(E_{t} \backslash E_{t+1} \mid E_{t}\right) \int_{E_{t+1}} u_{i}(s) p_{i}\left(d s \mid E_{t} \backslash E_{t+1}\right)+p_{i}\left(E_{t+1} \mid E_{t}\right)\left(V_{i, E_{t+1}}+c \frac{W_{\mathbf{u}_{-E_{t}}}}{p_{i}\left(E_{t+1} \mid E_{t}\right)}\right)\right)_{i \in I} .
\end{aligned}
$$

By Dynamic Consistency, this holds if and only if $U_{E_{t+1}}+c W_{\mathbf{u}_{-E_{t}}, \mathbf{u}_{E_{t} \backslash E_{t+1}}} \succsim_{\mathbf{u}_{-E_{t}}, \mathbf{u}_{E_{t} \backslash E_{t+1}}}$ $V_{E_{t+1}}+c W_{\mathbf{u}_{-E_{t}}, \mathbf{u}_{E_{t} \backslash E_{t+1}}}$, where $W_{\mathbf{u}_{-E_{t}}, \mathbf{u}_{E_{t} \backslash E_{t+1}}}=\left(W_{i, \mathbf{u}_{-E_{t}}} / p_{i}\left(E_{t+1} \mid E_{t}\right)\right)_{i \in I}$.

Theorem 10 For each fixed $t, E_{t}$ and $\mathbf{u}_{-E_{t}}$, the social welfare ordering $\succsim_{\mathbf{u}_{-E_{t}}}$ satisfies Order, Separability, EU-Pareto, EU-Continuity, EU-Inequality Aversion and EU-General Shift Covariance if and only if either of the following two cases holds:

(i) there exists a vector $\lambda\left(\mathbf{u}_{-E_{t}}\right) \in \mathbb{R}_{++}^{I}$ and a vector $a\left(\mathbf{u}_{-E_{t}}\right) \in \operatorname{int} \Delta(I)$ such that $\succsim_{\mathbf{u}_{-E_{t}}}$ is represented in the form

$$
\Phi\left(\mathbf{u}_{E_{t}} \mid \mathbf{u}_{-E_{t}}\right)=-\sum_{i \in I} a_{i}\left(\mathbf{u}_{-E_{t}}\right) e^{-\lambda_{i}\left(\mathbf{u}_{-E_{t}}\right) \int_{E_{t}} u_{i}(s) p_{i}\left(d s \mid E_{t}\right)}
$$

We call this class of orderings and representations generalized exponential class.

(ii) there exists a vector $a\left(\mathbf{u}_{-E_{t}}\right) \in$ int $\Delta(I)$ such that $\succsim_{\mathbf{u}_{-E_{t}}}$ is represented in the form

$$
\Phi\left(\mathbf{u}_{E_{t}} \mid \mathbf{u}_{-E_{t}}\right)=-\sum_{i \in I} a_{i}\left(\mathbf{u}_{-E_{t}}\right) \int_{E_{t}} u_{i}(s) p_{i}\left(d s \mid E_{t}\right)
$$

We call this class of orderings and representations additive class.

Moreover, for each $t$ and $\mathbf{u}_{-E_{t}}$, in case (i) $a\left(\mathbf{u}_{-E_{t}}\right)$ and $\lambda\left(\mathbf{u}_{-E_{t}}\right)$ are unique and in case (ii) $a\left(\mathbf{u}_{-E_{t}}\right)$ is unique.

Proof. It follows from the fact that $\succsim_{\mathbf{u}_{-E_{t}}}^{*}$ satisfies all the conditions in Lemma 2.

Now for the generalized exponential class we have

$$
\begin{aligned}
& \Phi\left(\mathbf{u}_{\Omega} \mid \emptyset\right) \\
= & -\sum_{i \in I} a_{i}(\emptyset) e^{-\lambda_{i}(\emptyset) \int_{\Omega} u_{i}(s) p_{i}(d s)} \\
= & -\sum_{i \in I} a_{i}(\emptyset) e^{-\lambda_{i}(\emptyset)\left(p_{i}\left(\Omega \backslash E_{t}\right) \int_{\Omega \backslash E_{t}} u_{i}(s) p_{i}\left(d s \mid \Omega \backslash E_{t}\right)+p_{i}\left(E_{t}\right) \int_{E_{t}} u_{i}(s) p_{i}\left(d s \mid E_{t}\right)\right)} \\
= & -\sum_{i \in I} a_{i}(\emptyset) e^{-\lambda_{i}(\emptyset) p_{i}\left(\Omega \backslash E_{t}\right) \int_{\Omega \backslash E_{t}} u_{i}(s) p_{i}\left(d s \mid \Omega \backslash E_{t}\right)} e^{-\lambda_{i}(\emptyset) p_{i}\left(E_{t}\right) \int_{E_{t}} u_{i}(s) p_{i}\left(d s \mid E_{t}\right)},
\end{aligned}
$$


which under Dynamic Consistency yields the same ranking over $\mathbf{u}_{E_{t}}$ as $\Phi\left(\mathbf{u}_{E_{t}} \mid \mathbf{u}_{-E_{t}}\right)=$ $-\sum_{i \in I} a_{i}\left(\mathbf{u}_{-E_{t}}\right) e^{-\lambda_{i}\left(\mathbf{u}_{-E_{t}}\right) \int_{E_{t}} u_{i}(s) p_{i}\left(d s \mid E_{t}\right)}$ does.

For the additive class, we have

$$
\begin{aligned}
& \Phi\left(\mathbf{u}_{\Omega} \mid \emptyset\right) \\
= & \sum_{i \in I} a_{i}(\emptyset) \int_{\Omega} u_{i}(s) p_{i}(d s) \\
= & \sum_{i \in I} a_{i}(\emptyset)\left(p_{i}\left(\Omega \backslash E_{t}\right) \int_{\Omega \backslash E_{t}} u_{i}(s) p_{i}\left(d s \mid \Omega \backslash E_{t}\right)+p_{i}\left(E_{t}\right) \int_{E_{t}} u_{i}(s) p_{i}\left(d s \mid E_{t}\right)\right) \\
= & \sum_{i \in I} a_{i}(\emptyset) p_{i}\left(\Omega \backslash E_{t}\right) \int_{\Omega \backslash E_{t}} u_{i}(s) p_{i}\left(d s \mid \Omega \backslash E_{t}\right)+\sum_{i \in I} a_{i}(\emptyset) p_{i}\left(E_{t}\right) \int_{E_{t}} u_{i}(s) p_{i}\left(d s \mid E_{t}\right),
\end{aligned}
$$

which under Dynamic Consistency yields the same ranking over $\mathbf{u}_{E_{t}}$ as $\Phi\left(\mathbf{u}_{E_{t}} \mid \mathbf{u}_{-E_{t}}\right)=$ $\sum_{i \in I} a_{i}\left(\mathbf{u}_{-E_{t}}\right) \int_{E_{t}} u_{i}(s) p_{i}\left(d s \mid E_{t}\right)$ does.

Therefore, after normalization, we obtain the following updating rule.

Theorem 11 The process of social welfare orderings $\left\{\succsim_{\mathbf{u}_{-E_{t}}}\right\}$ satisfies Order, Separability, EU-Pareto, EU-Continuity, EU-Inequality Aversion and EU-General Shift Covariance at $\emptyset$ and Dynamic Consistency if and only if either

(i) $\left\{\succsim_{\mathbf{u}_{-E_{t}}}\right\}$ falls in the generalized exponential class and $\left\{a\left(\mathbf{u}_{-E_{t}}\right)\right\}$ and $\left\{\lambda\left(\mathbf{u}_{-E_{t}}\right)\right\}$ follow the updating rule

$$
\begin{aligned}
a_{i}\left(\mathbf{u}_{-E_{t}}\right) & =\frac{a_{i}(\emptyset) e^{-\lambda_{i}(\emptyset) p_{i}\left(\Omega \backslash E_{t}\right) \int_{\Omega \backslash E_{t}} u_{i}(s) p_{i}\left(d s \mid \Omega \backslash E_{t}\right)}}{\sum_{j \in I} a_{j}(\emptyset) e^{-\lambda_{j}(\emptyset) p\left(\Omega \backslash E_{t}\right) \int_{\Omega \backslash E_{t}} u_{j}(s) p_{j}\left(d s \mid \Omega \backslash E_{t}\right)}} \\
\lambda_{i}\left(\mathbf{u}_{-E_{t}}\right) & =\lambda(\emptyset) p_{i}\left(E_{t}\right)
\end{aligned}
$$

for all $\mathbf{u}_{-E_{t}}$, or

(ii) $\left\{\succsim_{\mathbf{u}_{-E_{t}}}\right\}$ falls in the additive class and $\left\{a\left(\mathbf{u}_{-E_{t}}\right)\right\}$ follows

$$
a_{i}\left(\mathbf{u}_{-E_{t}}\right)=\frac{a_{i}(\emptyset) p_{i}\left(E_{t}\right)}{\sum_{j \in I} a_{j}(\emptyset) p_{j}\left(E_{t}\right)}
$$

for all $\mathbf{u}_{-E_{t}}$.

The theorem says that if the social ranking has equity concern it must incorporate utilities which turned out not to occur into account in updating welfare weights as described by formula (10), while the equity concern on each individual deceases probabilistically over 
time according to her belief as in formula (11). There, higher (lower) utilities which turned out not to occur must be 'compensated' in the way that they decrease (increase) welfare weights conditional on the present event. It also says that when the social ranking has no equity concern welfare weights conditional on an event must be proportional to her belief in the event as in formula (12), which depends on event occurred but does not take utilities which turned out not to occur into account.

This implies that when we require that social ranking should ignore utilities which turned out not to occur the only possibility is additive aggregation.

Corollary 6 Suppose that the process of social welfare orderings $\left\{\succsim_{\mathbf{u}_{-E_{t}}}\right\}$ satisfying Dynamic Consistency falls in the exponential class as characterized in Theorem 10 and 11. Then it satisfies Independence of Non-occurred Consequences hold if and only if it falls in the additive class with

$$
a_{i}\left(\mathbf{u}_{-E_{t}}\right)=\frac{a_{i}(\emptyset) p_{i}\left(E_{t}\right)}{\sum_{j \in I} a_{j}(\emptyset) p_{j}\left(E_{t}\right)}
$$

for all $\mathbf{u}_{-E_{t}}$.

Also, the above result implies that under heterogeneous beliefs the social ranking cannot be independent of events occurred. This is consistent with the result by Chambers and Hayashi [4] that under heterogeneous beliefs any social ranking respecting the Pareto principle cannot be state-independent.

Independence of Non-occurred Consequences and Events: for all $t$, for all $E_{t}$ and $\widetilde{E}_{t}$, for all $\mathbf{u}_{-E_{t}}$ and $\widetilde{\mathbf{u}}_{-\widetilde{E}_{t}}$, and for all $U, V \in \mathbb{R}^{I}$,

$$
\left(U_{i} \mathbf{1}_{E_{t}}\right)_{i \in I} \succsim_{\mathbf{u}_{-E_{t}}}\left(V_{i} \mathbf{1}_{E_{t}}\right)_{i \in I} \Longleftrightarrow\left(U_{i} \mathbf{1}_{\widetilde{E}_{t}}\right)_{i \in I} \succsim_{\widetilde{\mathbf{u}}_{-\widetilde{E}_{t}}}\left(V_{i} \mathbf{1}_{\widetilde{E}_{t}}\right)_{i \in I}
$$

Corollary 7 Under heterogeneous beliefs, there is no process of social welfare orderings which satisfies Dynamic Consistency and Independence of Non-occurred Consequences and Events.

\section{Conclusion}

We have argued that forward-lookingness, a central principle in economic decision making that a rational agent should be forward-looking and not be bound by things already gone, 
is not appealing for collective decision making, and that things already gone have necessary and substantive roles there.

Then we have considered a dynamic process of social welfare orderings explicitly, and propose a discipline which is acceptable even after rejecting the forward-lookingness principle. It is that the process must be dynamically consistent and a meaningful normative postulate should be invariant under consistent updating.

Based on this standpoint, we presented a set of axioms for social welfare orderings which are invariant to consistent updating, and characterize a set of social welfare functions which are closed under updating. With such a class of social welfare functions, we characterized the roles for pasts and things known not to occur, which are played in the updating stage.

\section{Appendix}

\section{A Proofs of Theorem 1 and 2}

\section{Proof of Theorem 1}

This is an asymmetric extension of the argument in Roberts ([13], Theorem 6) and Moulin ([11], Theorem 2.6).

Necessity of the axioms is routine. We prove sufficiency.

From Order, Continuity and Separability, $\succsim$ allows additive representation (see Debreu $[5])$

$$
\Phi(U)=\sum_{i \in I} \phi_{i}\left(U_{i}\right)
$$

which is unique positive affine transformations. From Pareto, each $\phi_{i}$ is strictly increasing.

From Shift Covariance, both $\sum_{i \in I} \phi_{i}\left(U_{i}\right)$ and $\sum_{i \in I} \phi_{i}\left(U_{i}+c\right)$ are additive representations of the same ranking, therefore cardinal equivalence: there exist functions $\psi$ and $\zeta$ with $\psi$ being positive such that

$$
\phi_{i}\left(U_{i}+c\right)=\psi(c) \phi_{i}\left(U_{i}\right)+\zeta(c)
$$

for all $i$.

This is generalized Pexider equation, which has a strongly increasing and weakly concave solution either in the form

$$
\phi_{i}\left(U_{i}\right)=-A_{i} e^{-\lambda_{i} U_{i}}
$$


with $A_{i}>$ and $\lambda_{i}>0$ or

$$
\phi_{i}\left(U_{i}\right)=A_{i} U_{i}
$$

with $A_{i}>0$.

Because $\phi$ and $\zeta$ are common across individuals, we must have

$$
\phi_{i}\left(U_{i}\right)=-A_{i} e^{-\lambda U_{i}}
$$

for all $i \in I$ or

$$
\phi_{i}\left(U_{i}\right)=A_{i} U_{i}
$$

for all $i \in I$.

By normalizing $a_{i}=A_{i} / \sum_{j \in I} A_{j}$ for each $i \in I$, we obtain the representation.

Uniqueness: On the exponential case, suppose both $-\sum_{i \in I} a_{i} e^{-\lambda U_{i}}$ and $-\sum_{i \in I} a_{i}^{\prime} e^{-\lambda^{\prime} U_{i}}$ represent the same ranking. Since they are additive representations of the same ranking, we have cardinal equivalence: there exist constants $C, D$ with $C>0$ such that

$$
-a_{i}^{\prime} e^{-\lambda^{\prime} U_{i}}=-C a_{i} e^{-\lambda U_{i}}+D
$$

for all $i$.

Suppose $D \neq 0$. Then as $U_{i} \rightarrow \infty$ we have $-a_{i}^{\prime} e^{-\lambda^{\prime} U_{i}} \rightarrow 0$ but $-C a_{i} e^{-\lambda U_{i}}+D \rightarrow D \neq 0$, a contradiction. Hence $D=0$.

By letting $U_{i}=0$ we have $a_{i}^{\prime}=C a_{i}$. Since $\sum_{i \in I} a_{i}=1$, we obtain $C=1$ which implies $a_{i}^{\prime}=a_{i}$. Then it is immediate to see $\lambda^{\prime}=\lambda$.

On the additive case, suppose both $\sum_{i \in I} a_{i} U_{i}$ and $\sum_{i \in I} a_{i}^{\prime} U_{i}$ represent the same ranking. Since they are additive representations of the same ranking, we have cardinal equivalence: there exist constants $C, D$ with $C>0$ such that

$$
-a_{i}^{\prime} U_{i}=-C a_{i} U_{i}+D
$$

for all $i$.

By letting $U_{i}$ we obtain $D=0$. By letting $U_{i}=1$ we have $C=1$ which implies $a_{i}^{\prime}=a_{i}$.

\section{Proof of Theorem 2}

Necessity of the axioms is routine. We prove sufficiency. 
Define $\succsim^{\star}$ by

$$
U \succsim^{\star} V \quad \Longleftrightarrow \quad\left(W_{i} U_{i} / \bar{W}\right)_{i \in I} \succsim\left(W_{i} V_{i} / \bar{W}\right)_{i \in I},
$$

where $\bar{W}=\sum_{i \in I} W_{i}$.

Then ${ }^{\star}$ satisfies Order, Continuity, Pareto, Shift Covariance and Separability, and follows from Theorem 1 that either of the following two cases holds;

(i) there exists $\lambda^{\star}>0$ and a vector $a^{\star} \in$ int $\Delta(I)$ such that $\succsim^{\star}$ is represented in the form

$$
\Phi^{\star}(U)=-\sum_{i \in I} a_{i}^{\star} e^{-\lambda^{\star} U_{i}}
$$

(ii) there exists a vector $a^{\star} \in$ int $\Delta(I)$ such that $\succsim$ is represented in the form

$$
\Phi^{\star}(U)=\sum_{i \in I} a_{i}^{\star} U_{i}
$$

In case (i), we have

$$
\begin{aligned}
U \succsim V & \Longleftrightarrow\left(U_{i} / W_{i}\right)_{i \in I} \succsim^{\star}\left(V_{i} / W_{i}\right)_{i \in I} \\
& \Longleftrightarrow-\sum_{i \in I} a_{i}^{\star} e^{-\lambda^{\star} \bar{W} U_{i} / W_{i}} \geq-\sum_{i \in I} a_{i}^{\star} e^{-\lambda^{\star} \bar{W} V_{i} / W_{i}}
\end{aligned}
$$

Therefore, by letting $\lambda_{i}=\lambda^{\star} \bar{W} / W_{i}$ for each $i \in I$ and $a=a^{\star}$, we obtain the representation. In case (ii), we have

$$
\begin{aligned}
U \succsim V & \Longleftrightarrow\left(\bar{W} U_{i} / W_{i}\right)_{i \in I} \succsim^{\star}\left(\bar{W} V_{i} / W_{i}\right)_{i \in I} \\
& \Longleftrightarrow \sum_{i \in I} a_{i}^{\star} \bar{W} U_{i} / W_{i} \geq-\sum_{i \in I} a_{i}^{\star} \bar{W} V_{i} / W_{i}
\end{aligned}
$$

Therefore, by letting $a_{i}=\frac{a_{i}^{\star} \bar{W} / W_{i}}{\sum_{j \in I} a_{j}^{\star} \bar{W} / W_{j}}$ for each $i \in I$ we obtain the representation.

Uniqueness: On the exponential case, suppose both $-\sum_{i \in I} a_{i} e^{-\lambda_{i} U_{i}}$ and $-\sum_{i \in I} a_{i}^{\prime} e^{-\lambda_{i}^{\prime} U_{i}}$ represent the same ranking. Since they are additive representations of the same ranking, we have cardinal equivalence: there exist constants $C>0$ and $\left(D_{i}\right)_{i \in I}$ such that

$$
-a_{i}^{\prime} e^{-\lambda_{i}^{\prime} U_{i}}=-C a_{i} e^{-\lambda_{i} U_{i}}+D_{i}
$$

for all $i$.

Suppose $D_{i} \neq 0$. Then as $U_{i} \rightarrow \infty$ we have $-a_{i}^{\prime} e^{-\lambda_{i}^{\prime} U_{i}} \rightarrow 0$ but $-C a_{i} e^{-\lambda_{i} U_{i}}+D_{i} \rightarrow$ $D_{i} \neq 0$, a contradiction. Hence $D_{i}=0$.

By letting $U_{i}=0$ we have $a_{i}^{\prime}=C a_{i}$. Since $\sum_{i \in I} a_{i}=1$, we obtain $C=1$ which implies $a_{i}^{\prime}=a_{i}$. Then it is immediate to see $\lambda_{i}^{\prime}=\lambda_{i}$, which is true for all $i$.

Uniqueness for the additive case is immediate. 


\section{References}

[1] Elchanan Ben-Porath, Itzhak Gilboa and David Schmeidler (1997): On the Measurement of Inequality under Uncertainty, Journal of Economic Theory, 75, 194-204.

[2] Antoine Bommier and Stephane Zuber (2010): Inequality Aversion and Time Consistency, working paper, Toulouse School of Economics.

[3] Margaret Wise Brown (1993): Walt Disney's: The Grasshopper and the Ants, Disney Press, New York.

[4] Christopher P. Chambers and Takashi Hayashi (2009): Preference aggregation under uncertainty: Savage vs. Pareto, Games and Economic Behavior, Vol 54, Issue 2, February 2006, 430-440.

[5] Gerard Debreu (1960): Topological Methods in Cardinal Utility Theory, in Arrow, Karlin and Suppes, editors, Mathematical Methods in the Social Sciences, Stanford University Press, pp. 16-26.

[6] Peter A. Diamond (1967): Cardinal Welfare, Individualistic Ethics, and Interpersonal Comparisons of Utility: Comment, Journal of Political Economy, 75: 765-766.

[7] Larry Epstein and Uzi Segal (1992): Quadratic Social Welfare Functions, Journal of Political Economy, 100: 691-712.

[8] Peter Hammond (1996): Consequentialist Decision Theory and Utilitarian Ethics in F. Farina, F. Hahn, and S. Vannucci (eds.) Ethics, Rationality, and Economic Behaviour, Oxford: Clarendon Press, pp. 92-118.

[9] John C. Harsanyi (1955): Cardinal Welfare, Individualistic Ethics, and Interpersonal Comparisons of Utility, Journal of Political Economy, 63: 309-321.

[10] Mark J. Machina (1989): Dynamic Consistency and Non-expected Utility Models of Choice under Uncertainty, Journal of Economic Literature, vol. 27(4), pages 1622-68.

[11] Herve Moulin (1989): Axioms of Cooperative Decision Making, Cambridge: Cambridge University Press. 
[12] Ben Edwin Perry (1965): Babrius and Phaedrus / newly edited and translated into English, together with an historical introduction and a comprehensive survey of Greek and Latin fables in the Aesopic tradition, W. Heinemann ; Harvard University Press, London : Cambridge, Mass.

[13] Kevin Roberts (1980): Interpersonal Comparability and Social Choice Theory, Review of Economic Studies, vol. 47, issue 2, pages 421-39.

[14] Stephane Zuber (2010): The aggregation of preferences: can we ignore the past?, Theory and Decision, forthcoming. 